\title{
Separable Potentials and a Triality in Two-Dimensional Spaces of Constant Curvature
}

\author{
José F. CARINENA ${ }^{\dagger}$, Manuel F RAÑADA ${ }^{\dagger}$, Mariano SANTANDER \\ and Teresa SANZ-GIL $\ddagger$ \\ $\dagger$ Departamento de Física Teórica, Facultad de Ciencias, Universidad de Zaragoza \\ 50009 Zaragoza, Spain \\ E-mail: jfc@posta.unizar.es,mfran@unizar.es \\ ‡ Departamento de Física Teórica, Universidad de Valladolid \\ 47011 Valladolid, Spain \\ E-mail:msn@fta.uva.es,mtsanz@fta.uva.es
}

Received December, 2004; Accepted February, 2005

\begin{abstract}
We characterize and completely describe some types of separable potentials in twodimensional spaces, $S_{\left[\kappa_{1}\right] \kappa_{2}}^{2}$, of any (positive, zero or negative) constant curvature and either definite or indefinite signature type. The results are formulated in a way which applies at once for the two-dimensional sphere $\mathbf{S}^{2}$, hyperbolic plane $\mathbf{H}^{2}$, AntiDeSitter / DeSitter two-dimensional spaces $\mathbf{A} \mathbf{d} \mathbf{S}^{1+1} / \mathbf{d} \mathbf{S}^{1+1}$ as well as for their flat analogues $\mathbf{E}^{2}$ and $\mathbf{M}^{1+1}$. This is achieved through an approach of Cayley-Klein type with two parameters, $\kappa_{1}$ and $\kappa_{2}$, to encompass all curvatures and signature types. We discuss six coordinate systems allowing separation of the Hamilton-Jacobi equation for natural Hamiltonians in $S_{\left[\kappa_{1}\right] \kappa_{2}}^{2}$ and relate them by a formal triality transformation, which seems to be a clue to introduce general "elliptic coordinates" for any CK space concisely. As an application we give, in any $S_{\left[\kappa_{1}\right] \kappa_{2}}^{2}$, the explicit expressions for the Fradkin tensor and for the Runge-Lenz vector, i.e., the constants of motion for the harmonic oscillator and Kepler potential on any $S_{\left[\kappa_{1}\right] \kappa_{2}}^{2}$.
\end{abstract}

\section{Introduction}

The aim of this paper is to characterize and completely describe some types of separable potentials in $2 \mathrm{~d}$ spaces of constant curvature and either Riemannian or Lorentzian type metric using a unified and joint description. All these spaces are members of the two parametric family of Cayley-Klein (CK) two-dimensional spaces, with the parameters $\kappa_{1}, \kappa_{2}$ corresponding to the curvature of the space and to the signature type. All the results obtained by such procedure make sense for the whole family of spaces with any constant curvature (no matter positive, zero or negative) and any metric signature, no matter definite (Riemannian) or indefinite (pseudoRiemannian Lorentzian type).

Separation of variables in the Hamilton-Jacobi equation has been discussed in the literature for some individual spaces on a case by case basis. The situation has been quite 
exhaustively studied when configuration space is Euclidean $\mathbf{E}^{2}$, starting in [3]. This has been also extended to systems on the sphere $\mathbf{S}^{2}$ or on the hyperbolic plane $\mathbf{H}^{2}[4,5,6$, $7,13,14]$. The case where configuration space has an Lorentzian (indefinite) constant curvature metric is less known, albeit Drach potentials are linked to the Minkowski space $[20,22]$. A list of inequivalent quadratically superintegrable systems in $\mathbf{M}^{1+1}$ exists [13] yet its interpretation in terms of generic 'elliptic' coordinates in Minkowski space and their limiting or special cases is still not fully understood. We do not know any previous study neither for AntiDeSitter $\mathbf{A} \mathbf{d} \mathbf{S}^{1+1}$ or the DeSitter $\mathbf{d} \mathbf{S}^{1+1}$ configuration spaces, nor a study of their relation through contraction to the superintegrable systems in $\mathbf{M}^{1+1}$.

Why is this kind of approach worthwhile? From a more practical side, approaching the problem on a case-by-case basis must be supplemented with a study of the limiting transitions where, for instance, curvature vanishes. This need can be dispensed provided we instead look to all cases as members of a parametrised full family of spaces with every detail arranged so as to have limiting situations (vanishing curvature $\kappa_{1}=0$ and/or degenerating metric $\kappa_{2}=0$ ) described smoothly at the same level as the generic ones. In papers $[21,23,24,25,26]$ this is done for $\kappa_{2}=1$ and only a curvature parameter $\kappa=\kappa_{1}$. From a more structural perspective the approach we are proposing makes some essential aspects to stand out clearly and put to the forefront a deep analogy between all the cases (any $\kappa_{1}, \kappa_{2}$ ), while a case by case approach risks to highlight some non essential differences while hiding some essential similarities.

We deal with a system with a Lagrangian of mechanical type, i.e., a "kinetical" term minus a potential function, in a $2 \mathrm{~d}$ space $S_{\left[\kappa_{1}\right] \kappa_{2}}^{2}$ of constant curvature $\kappa_{1}$ and metric with signature $\left(+, \kappa_{2}\right)$. When is the corresponding Hamilton-Jacobi equation separable? For $\mathbf{S}^{2}$ the answer has been long known: the potential $V$ must be separable in Jacobi elliptic coordinates, which are determined by two points (the focal points) on $\mathbf{S}^{2}$; the coordinates themselves are (half) the sum and the difference of geodesic distances from the generic point $P$ to the two foci; these potentials allow for an extra constant of motion further to the energy. The situation in $\mathbf{H}^{2}$ and in AntiDeSitter and DeSitter spaces $\mathbf{A d} \mathbf{S}^{1+1}, \mathbf{d} \mathbf{S}^{1+1}$ is apparently more complicated and, if elliptic is taken in a literal sense of (half) sum and difference of geodesic distances from the generic point $P$ to the two foci, then definitely there are other coordinate systems allowing separation of variables in the Hamilton-Jacobi equation in $\mathbf{H}^{2}$. With the right interpretation, however, all these systems should be understood as general elliptic, in the sense of confocal conics, yet the foci are not necessarily proper points in $S_{\left[\kappa_{1}\right] \kappa_{2}}^{2}[27]$. In this paper we characterize, for all values of $\kappa_{1}$ and $\kappa_{2}$, hence in all CK $2 \mathrm{~d}$ spaces, several sets of potentials having extra quadratic constants of motion and separable in some specific coordinate systems, which also allow Hamilton-Jacobi separation. This should be taken as a first step towards the full characterization of separable potentials in $2 \mathrm{~d}$ configuration spaces of constant curvature and any signature; it displays in the simplest situations the traits of the $\kappa_{1}, \kappa_{2}$ parametric approach. The triality idea we introduce here is also essential to get the classification of superintegrable systems bypassing the need of brute-force direct calculations.

The paper is arranged as follows. First we describe the required basics of the CK type approach. Then we introduce six particular coordinate systems and give explicitly all expressions pertinent for the geometry of $S_{\left[\kappa_{1}\right] \kappa_{2}}^{2}$ in their terms. In the next section potentials separable in these six coordinates systems are shown to have an extra constant of motion additional to the "energy", which is also given in explicit form; these systems 
allow Hamilton-Jacobi separation of variables for any value of $\kappa_{1}, \kappa_{2}$. Then we give a brief statement of the basic triality which underlies most of the paper. To close we discuss some interesting examples and give, in any $S_{\left[\kappa_{1}\right] \kappa_{2}}^{2}$, explicit expressions for the constants of motion specific to the "curved potentials" analogues to harmonic oscillator (the so called Fradkin tensor) and to the Kepler problem (the Laplace-Runge-Lenz vector) in any $S_{\left[\kappa_{1}\right] \kappa_{2}}^{2}$.

\section{The geometry of $S_{\left[\kappa_{1}\right] \kappa_{2}}^{2}$}

We first introduce the necessary details on the $2 \mathrm{~d}$ spaces $S_{\left[\kappa_{1}\right] \kappa_{2}}^{2}$ with constant curvature $\kappa_{1}$ and metric of signature type $\left(+, \kappa_{2}\right)$ (for more details and some applications see $[8,9,10]$ ). These are homogeneous spaces. Hence they admit a maximal three-dimensional isometry group, which we call $S O_{\kappa_{1}, \kappa_{2}}(3)$ and which is generated by a three dimensional Lie algebra $s o_{\kappa_{1}, \kappa_{2}}(3)$, given in a matrix realization as:

$$
P_{1}=\left(\begin{array}{ccc}
0 & -\kappa_{1} & 0 \\
1 & 0 & 0 \\
0 & 0 & 0
\end{array}\right) \quad P_{2}=\left(\begin{array}{ccc}
0 & 0 & -\kappa_{1} \kappa_{2} \\
0 & 0 & 0 \\
1 & 0 & 0
\end{array}\right) \quad J=\left(\begin{array}{ccc}
0 & 0 & 0 \\
0 & 0 & -\kappa_{2} \\
0 & 1 & 0
\end{array}\right),
$$

where we stress the association among generators $P_{1}, P_{2}$ and $J$ and constants $\kappa_{1}, \kappa_{1} \kappa_{2}$ and $\kappa_{2}$. The commutators between the generators $J, P_{1}$ and $P_{2}$ are:

$$
\left[J, P_{1}\right]=P_{2} \quad\left[J, P_{2}\right]=-\kappa_{2} P_{1} \quad\left[P_{1}, P_{2}\right]=\kappa_{1} J .
$$

This approach embodies essentially nine cases, because by scaling each of the constants $\kappa_{1}, \kappa_{2}$ may be brought to their standard values $1,0,-1$; the essential information is whether $\kappa_{1}\left(\right.$ resp. $\left.\kappa_{2}\right)$ are positive, zero or negative, and this corresponds to the elliptic/parabolic/hyperbolic character of the measure of lenghts (resp. angles) within the formalism of projective metrics. This is depicted in Table 1.

Each entry of the Table gives the name of each homogeneous space $G / H$, its isometry group $G$ and the isotropy subgroup $H$. The three rows accomodate spaces with either a Riemannian, degenerate Riemannian and pseudoRiemannian (Lorentzian) metric, according to the sign of $\kappa_{2}$, and the three instances along each row correspond to spaces with constant positive, zero or negative curvature. There are five non-isomorphic isometry groups, two generic simple ones $S O(3), S O(2,1)$, two limiting 1-quasisimple ones $I S O(2), I S O(1,1)$ and one doubly limiting, 2-quasisimple, the Galilei group IISO(1), isomorphic to the Heisenberg group. As far as the homogeneous spaces themselves, there are eight different ones; in 2d the DeSitter and AntiDeSitter spaces only differ by the change of the sign in the metric and in this sense they might be considered as isomorphic spaces.

It may be relevant to remark that for $G=S O(3)$ we have a single homogeneous space of CK type associated to $G$, because all possible subgroups $H$ are conjugate. The other simple CK group $S O(2,1)$ has three associated homogeneous spaces which, albeit linked by duality and by change of sign in the Lorentzian metric, should be considered as inequivalent from the CK viewpoint. For instance, $\mathbf{H}^{2}$ and DeSitter/AntiDeSitter space have the same isometry group $S O(2,1)$, but their isotopy subgroups $S O(2)$ and $S O(1,1)$ are non conjugated. While $\mathbf{H}^{2}$ is a space with Riemannian metric and negative constant curvature, $\mathbf{A} \mathbf{d} \mathbf{S}^{1+1}$ or $\mathbf{d} \mathbf{S}^{1+1}$ are constant curvature spaces with a Lorentzian metric; these three spaces are related by a triality [28], a formal transformation which is not an isometry 
Table 1. The nine two-dimensional CK spaces $S_{\left[\kappa_{1} \mid \kappa_{2}\right.}^{2}$.

\begin{tabular}{|c|c|c|c|}
\hline \multirow[b]{2}{*}{$\begin{array}{l}\text { Measure of angle } \\
\& \text { Sign of } \kappa_{2}\end{array}$} & \multicolumn{3}{|c|}{ Measure of distance \& Sign of $\kappa_{1}$} \\
\hline & $\begin{array}{l}\text { Elliptic } \\
\kappa_{1}=1\end{array}$ & $\begin{array}{l}\text { Parabolic } \\
\kappa_{1}=0\end{array}$ & $\begin{array}{l}\text { Hyperbolic } \\
\kappa_{1}=-1\end{array}$ \\
\hline $\begin{array}{l}\text { Elliptic } \\
\kappa_{2}=1\end{array}$ & $\begin{array}{l}\text { Elliptic } \\
G=S O(3) \\
H=S O(2) \\
G / H=\mathbf{S}^{2}\end{array}$ & $\begin{array}{l}\text { Euclidean } \\
G=I S O(2) \\
H=S O(2) \\
G / H=\mathbf{E}^{2}\end{array}$ & $\begin{array}{l}\text { Hyperbolic } \\
G=S O(2,1) \\
H=S O(2) \\
G / H=\mathbf{H}^{2}\end{array}$ \\
\hline $\begin{array}{l}\text { Parabolic } \\
\kappa_{2}=0\end{array}$ & $\begin{array}{l}\text { Co-Euclidean } \\
\text { Oscillating NH } \\
G=I S O(2) \\
H=\mathbb{R} \\
G / H=\mathbf{A N H}^{1+1}\end{array}$ & $\begin{array}{l}\text { Galilean } \\
\begin{array}{l}G=I I S O(1) \\
H=\mathbb{R} \\
G / H=\mathbf{G}^{1+1}\end{array}\end{array}$ & $\begin{array}{l}\text { Co-Minkowskian } \\
\text { Expanding NH } \\
G=I S O(1,1) \\
H=\mathbb{R} \\
G / H=\mathbf{N H}^{1+1}\end{array}$ \\
\hline $\begin{array}{l}\text { Hyperbolic } \\
\kappa_{2}=-1\end{array}$ & $\begin{array}{l}\text { Co-Hyperbolic } \\
\text { Anti-de Sitter } \\
G=S O(2,1) \\
H=S O(1,1) \\
G / H=\mathbf{A d} \mathbf{S}^{1+1}\end{array}$ & $\begin{array}{l}G=I S O(1,1) \\
H=S O(1,1) \\
G / H=\mathbf{M}^{1+1}\end{array}$ & $\begin{array}{l}\text { Doubly Hyperbolic } \\
\text { De Sitter } \\
G=S O(2,1) \\
H=S O(1,1) \\
G / H=\mathbf{d S}^{1+1}\end{array}$ \\
\hline
\end{tabular}

in these three cases, but reduces to an isometry - the 3-fold rotation around the center of 'an octant' spherical triangle - of the sphere $\mathbf{S}^{2}$.

From the previous description and from Table 1, it follows that when both constants are positive, the space $S_{\left[\kappa_{1}\right] \kappa_{2}}^{2}$ is the two-dimensional sphere; the standard $\mathbf{S}^{2}$ corresponds to $\kappa_{1}=1, \kappa_{2}=1$. In this case $(2.2)$ reduces to the $s o(3)$ usual commutation relations. In the Euclidean case $\kappa_{1}=0, \kappa_{2}>0$, which can be reduced to the standard Euclidean space $\mathbf{E}^{2}\left(\kappa_{1}=0, \kappa_{2}=1\right)$, the commutators (2.2) close iso(2) commutation relations, with commuting translations and $\left(P_{1}, P_{2}\right)$ behaving as an so(2)-vector under rotations. When $\kappa_{1}<0, \kappa_{2}>0$ we get Lobachevski hyperbolic space, $\mathbf{H}^{2}$ whose isometry algebra $s o_{-1,1}(3)$ is isomorphic to $s o(2,1)$. When $\kappa_{2}<0$, the commutation relations show that $\left(P_{1}, P_{2}\right)$ is a Lorentzian $s o(1,1)$-vector under the rotations generated by $J$ (now hyperbolic type) and the translations generators commute to a multiple of $J$ according to the sign of $\kappa_{1}$. Minkowskian geometry appears for $\kappa_{1}=0, \kappa_{2}<0$; if conventional measures are used for the time $P_{1}$ and space $P_{2}$ translations, then $\kappa_{2}=-1 / c^{2}$ in terms of the relativistic constant and the algebra $(2.2)$ close the familiar Poincaré $i s o(1,1)$ commutation relations in $1+1$ dimensions.

We now introduce the $\kappa$-Cosine $C_{\kappa}(x)$ l, Sine $S_{\kappa}(x)$ and Tangent $T_{\kappa}(x)$ functions:

$$
C_{\kappa}(x):=\left\{\begin{array}{ll}
\cos \sqrt{\kappa} x \\
1 \\
\cosh \sqrt{-\kappa} x
\end{array}, \quad S_{\kappa}(x):=\left\{\begin{array}{ll}
\frac{1}{\sqrt{\kappa}} \sin \sqrt{\kappa} x & \kappa>0 \\
x & \kappa=0 \\
\frac{1}{\sqrt{-\kappa}} \sinh \sqrt{-\kappa} x & \kappa<0
\end{array} \quad T_{\kappa}(x):=\frac{S_{\kappa}(x)}{C_{\kappa}(x)} .\right.\right.
$$

These functions include trigonometric $\kappa>0$ and hyperbolic $\kappa<0$ functions; we refer to [8] and [9] for further details on this $\kappa_{1}, \kappa_{2}$ formalism. When $\kappa=0$, the cosine reduces to the constant function 1 and the sine and tangent are the identity linear function of their variables.

Exponentials of matrices (2.1) lead to one-parametric subgroups $\exp \left(\alpha P_{1}\right), \exp \left(\beta P_{2}\right)$, 
$\exp (\gamma J)$ of $S O_{\kappa_{1}, \kappa_{2}}(3)$ :

$$
\begin{aligned}
& \exp \left(\alpha P_{1}\right)=\left(\begin{array}{ccc}
C_{\kappa_{1}}(\alpha) & -\kappa_{1} S_{\kappa_{1}}(\alpha) & 0 \\
S_{\kappa_{1}}(\alpha) & C_{\kappa_{1}}(\alpha) & 0 \\
0 & 0 & 1
\end{array}\right), \quad \exp \left(\gamma J_{12}\right)=\left(\begin{array}{ccc}
1 & 0 & 0 \\
0 & C_{\kappa_{2}}(\gamma) & -\kappa_{2} S_{\kappa_{2}}(\gamma) \\
0 & S_{\kappa_{2}}(\gamma) & C_{\kappa_{2}}(\gamma)
\end{array}\right) \\
& \exp \left(\beta P_{2}\right)=\left(\begin{array}{ccc}
C_{\kappa_{1} \kappa_{2}}(\beta) & 0 & -\kappa_{1} \kappa_{2} S_{\kappa_{1} \kappa_{2}}(\beta) \\
0 & 1 & 0 \\
S_{\kappa_{1} \kappa_{2}}(\beta) & 0 & C_{\kappa_{1} \kappa_{2}}(\beta)
\end{array}\right) .
\end{aligned}
$$

Now the matrix group $S O_{\kappa_{1}, \kappa_{2}}(3)$ acts by matrix multiplication on an $\mathbb{R}^{3}$ ambient space by isometries of the "ambient space metric":

$$
d s^{2}=\left(d s^{0}\right)^{2}+\kappa_{1}\left(d s^{1}\right)^{2}+\kappa_{1} \kappa_{2}\left(d s^{2}\right)^{2},
$$

and the space $S_{\left[\kappa_{1}\right] \kappa_{2}}^{2}$ is the coset space $S O_{\kappa_{1}, \kappa_{2}}(3) / S O_{\kappa_{2}}(2)$, where $S O_{\kappa_{2}}(2)=\langle J\rangle$. The space $S_{\left[\kappa_{1} \kappa_{2}\right.}^{2}$ can be described as the orbit $\Sigma \equiv\left(s^{0}\right)^{2}+\kappa_{1}\left(s^{1}\right)^{2}+\kappa_{1} \kappa_{2}\left(s^{2}\right)^{2}=1$ of the point $\left(s^{0}, s^{1}, s^{2}\right)=(1,0,0)$ under the group action. When $\kappa_{1} \neq 0$, the natural metric on $S_{\left[\kappa_{1}\right] \kappa_{2}}^{2}$, which will be always denoted $\mathbf{g}$, is obtained from the metric induced on the orbit by the CK ambient space metric as:

$$
\mathbf{g} \equiv g_{\mu \nu}\left(q^{1}, q^{2}\right) d q^{\mu} d q^{\nu}=d l^{2}=\left.\frac{1}{\kappa_{1}} d s^{2}\right|_{\Sigma},
$$

where, contrary to appearances, $d l^{2}$ has a well defined limit when $\kappa_{1} \rightarrow 0$. With this metric, the scheme includes simultaneously the four well known realizations of:

- the standard sphere $\mathbf{S}^{2}$ with the Riemannian metric of curvature 1 as the submanifold $\left(s^{0}\right)^{2}+\left(s^{1}\right)^{2}+\left(s^{2}\right)^{2}=1$ of the ambient three-dimensional Euclidean space with $d s^{2}=$ $d\left(s^{0}\right)^{2}+d\left(s^{1}\right)^{2}+d\left(s^{2}\right)^{2}$

- the standard hyperbolic plane $\mathbf{H}^{2}$ with the Riemannian metric of curvature -1 as the submanifold $\left(s^{0}\right)^{2}-\left(s^{1}\right)^{2}-\left(s^{2}\right)^{2}=1$ of the ambient three-dimensional Minkowskian space $d s^{2}=d\left(s^{0}\right)^{2}-d\left(s^{1}\right)^{2}-d\left(s^{2}\right)^{2}$; note that $\mathbf{g}$ is directly definite positive here.

- the AntiDeSitter sphere $\mathbf{A} \mathbf{d} \mathbf{S}^{1+1}$ with the pseudoRiemannian metric of curvature 1 as the submanifold $\left(s^{0}\right)^{2}+\left(s^{1}\right)^{2}-\left(s^{2}\right)^{2}=1$ of an ambient three-dimensional Minkowskian space with $d s^{2}=d\left(s^{0}\right)^{2}+d\left(s^{1}\right)^{2}-d\left(s^{2}\right)^{2}$, and finally

- the DeSitter sphere $\mathbf{d S}^{1+1}$ with the pseudoRiemannian metric of curvature -1 as the submanifold $\left(s^{0}\right)^{2}-\left(s^{1}\right)^{2}+\left(s^{2}\right)^{2}=1$ of an ambient three-dimensional Minkowskian space with $d s^{2}=d\left(s^{0}\right)^{2}-d\left(s^{1}\right)^{2}+d\left(s^{2}\right)^{2}$.

Spaces with vanishing curvature (as $\mathbf{E}^{2}, \mathbf{M}^{1+1}$ ) are described here as particular cases: $\mathbf{E}^{2}$ corresponds to $\kappa_{1}=0, \kappa_{2}=1$, and $\mathbf{M}^{1+1}$ corresponds to $\kappa_{1}=0, \kappa_{2}=-1$, so the formalism has built-in the pertinent contractions. Hence in this CK approach all expressions, results, etc. implicitly depend upon the parameters $\kappa_{1}, \kappa_{2}$ in such a way that particularizing them to some values will always lead meaningfully to the corresponding geometry without any need for a limiting procedure or contraction.

\section{Six coordinate systems}

We consider in this paper six particular coordinate systems on $S_{\left[\kappa_{1}\right] \kappa_{2}}^{2}$. All these turn out to be the simplest particular instances (with zero values for the interfocal distances) of elliptic, 
parabolic and ultraelliptic type coordinate systems in $S_{\left[\kappa_{1}\right] \kappa_{2}}^{2}[27]$. A complete discussion of the "general elliptic" coordinates in these spaces will be done elsewhere. Here we restrict ourselves to a self-contained presentation of these six coordinate systems with the aim to introduce a tool, the $T$-symmetry, which is essential to perform a meaningful comparison between Jacobi elliptic coordinates on the sphere and the more general "elliptic" coordinate systems allowing Hamilton-Jacobi separation of variables in $S_{\left[\kappa_{1}\right] \kappa_{2}}^{2}$.

These six coordinates systems are built in any of the CK spaces out of canonical parameters of suitable transformations (rotations around a fixed point $O$ and translations along two mutually intersecting orthogonal (oriented and cooriented) straight lines $l_{1}, l_{2}$ through $O)$. In all cases we take $O \equiv(1,0,0)$ on the $s^{0}$ axis and $l_{1}$ (resp. $l_{2}$ ) are the intersections of the CK sphere $\Sigma$ with the 2-planes $s^{0} s^{1}\left(\operatorname{resp} . s^{0} s^{2}\right)$ in the ambient CK space. The line $l_{1}$ has a tangent vector with positive square length $g_{\mu \nu} q^{\mu} q^{\nu}>0$, but the square length of the tangent vector to $l_{2}$ has the same sign as $\kappa_{2}$; then when $\kappa_{2}<0 l_{1}$ and $l_{2}$ are two lines of different type: in the language of relativity $l_{1}$ is time-like and $l_{2}$ is space-like. Therefore lengths along $l_{2}$ computed for the intrinsic metric $\mathbf{g}$ of $S_{\left[\kappa_{1}\right] \kappa_{2}}^{2}$ are pure imaginary and only become real for the associated metric $\mathbf{g} / \kappa_{2}$ (this is tantamount to the relation between metrics determining directly proper time and proper length in relativity).

Parameters for the translations $\exp \left(\alpha P_{1}\right)$ along $l_{1}$ carry a 'label' $\kappa_{1}$; these coincide to the distances - after $\mathbf{g}$ - along $l_{1}$ between any point on $l_{1}$ and its image under $\exp \left(\alpha P_{1}\right)$.

Parameters of the translations $\exp \left(\beta P_{2}\right)$ along $P_{2}$, carry a 'label' $\kappa_{1} \kappa_{2}$. The 'distance' -after $\mathbf{g}$ - along the line $l_{2}$ between any point on $l_{2}$ and its image equals $\sqrt{\kappa_{2}} \beta$, hence these 'distances' differ from the canonical parameter $\beta$ just by a constant factor which becomes pure imaginary when the metric is Lorentzian type. The quantity $\sqrt{\kappa_{2}} \beta$ should be considered as having a label $\kappa_{1}$; multiplication by $\sqrt{\kappa_{2}}$ serves to transfer the quantity $\beta$ with label $\kappa_{1} \kappa_{2}$ to another quantity $\sqrt{\kappa_{2}} \beta$ with label $\kappa_{1}$. Of course, these and similar transfers require $\kappa_{1}, \kappa_{2}$ constants to be different from zero. While transfers by themselves do not apply for the nongeneric spaces in the CK family, when combined with the triality to be discussed later they produce a result which is well defined for any value, even zero, of $\kappa_{1}, \kappa_{2}$.

Finally parameters for rotations around $O$ carry 'label' $\kappa_{2}$ and they coincide with the angle between any line through $O$ and its rotated, again determined from $\mathbf{g}$ as usual.

Now we define, for any generic point $P$, six quantities $r, \phi ; u, y ; x, v$ so that the point $P$ can be obtained as the image of $O$ by some products of one-parameter subgroups:

$$
P=\exp (\phi J) \exp \left(r P_{1}\right) O=\exp \left(u P_{1}\right) \exp \left(y P_{2}\right) O=\exp \left(v P_{2}\right) \exp \left(x P_{1}\right) O
$$

Note that the six quantities, to be taken as coordinates, do not appear primarily as lengths but instead as canonical parameters and each one has a label, which would fit to the label of the functions appearing in the associated isometries in (3.1) according to

$$
r \leftrightarrow \kappa_{1}, \quad \phi \leftrightarrow \kappa_{2} ; \quad u \leftrightarrow \kappa_{1}, \quad y \leftrightarrow \kappa_{1} \kappa_{2} ; \quad x \leftrightarrow \kappa_{1}, \quad v \leftrightarrow \kappa_{1} \kappa_{2} .
$$

Their precise relation to the lengths as computed from the metric $\mathbf{g}$ is:

- $r$ is the length from $P$ the origin $O$, measured along the line $O P$.

- $\phi$ is the angle between the positive half-ray of $l_{1}$ and the straight line $O P$ through $O$.

- $x$ is the length from $P$ to $l_{2}$, measured along a straight line $P_{2} P$ which is orthogonal to $l_{2}\left(P_{2}\right.$ is the orthogonal projection of $P$ on $\left.l_{2}\right)$; this line can be obtained from $l_{1}$ by a translation $\exp \left(v P_{2}\right)$ so that this is consistent with $x$ having label $\kappa_{1}$. 
- $v$ is only "proportional" to the length between $O$ and $P_{2}$ along $l_{2}$, with a factor $\sqrt{\kappa_{2}}$, so the length itself is $\sqrt{\kappa_{2}} v$. This is consistent with $v$ having the label $\kappa_{1} \kappa_{2}$; alternatively $v$ can be considered as the "space-like" distance measured with the metric $\mathbf{g} / \kappa_{2}$.

- $y$ is again only "proportional" to the length from $P$ to $P_{1}$ along the perpendicular to $l_{1}$ through $P$, which intersects $l_{1}$ at the point $P_{1}$. The length is equal to $\sqrt{\kappa_{2}} y$ so that this is consistent with $y$ having the label $\kappa_{1} \kappa_{2}$; alternatively $y$ can be considered as the "space-like"-length measured with the metric $\mathbf{g} / \kappa_{2}$ along the line from $P_{1}$ to $P$.

- Finally $u$ is the length between $O$ and $P_{1}$ along $l_{1}$, which coincides with the canonical parameter; this is consistent with $u$ having the label $\kappa_{1}$.

Now we introduce the coordinate systems themselves. These come in two groups of three systems, the mutual relationships of which are discussed later. Three coordinate systems, called polar, parallel '1' and parallel '2', are the particularization to the spaces $S_{\left[\kappa_{1}\right] \kappa_{2}}^{2}$ of the systems known as geodesic polar coordinates and geodesic parallel (or Gaussian) coordinates in differential geometry of surfaces. The remaining three coordinate systems will be called equiparabolic '01', equiparabolic '20', and equiparabolic '12'. They are also distinguished particular instances of the "general elliptic" coordinate systems on $S_{\left[\kappa_{1}\right] \kappa_{2}}^{2}[27]$, but here they are introduced directly from their expressions.

\subsection{Polar coordinates}

The coordinates are $(r, \phi)$. By direct substitution of the isometries in the definition of coordinates we get:

$$
\left(\begin{array}{c}
s^{0} \\
s^{1} \\
s^{2}
\end{array}\right)=\left(\begin{array}{c}
C_{\kappa_{1}}(r) \\
S_{\kappa_{1}}(r) C_{\kappa_{2}}(\phi) \\
S_{\kappa_{1}}(r) S_{\kappa_{2}}(\phi)
\end{array}\right), \quad d l^{2}=d r^{2}+\kappa_{2} S_{\kappa_{1}}^{2}(r) d \phi^{2},
$$

where the metric $\mathbf{g}$ reduces to $d r^{2}+\sin ^{2} r d \phi^{2}, d r^{2}+r^{2} d \phi^{2}, d r^{2}+\sinh ^{2} r d \phi^{2}$ for the three standard spaces $\mathbf{S}^{2}, \mathbf{E}^{2}, \mathbf{H}^{2}$, and to their Minkowskian versions, with opposite sign in the terms in $d \phi^{2}$, for the three pseudoRiemannian spaces $\mathbf{A d} \mathbf{S}^{1+1}, \mathbf{M}^{1+1}, \mathbf{d} \mathbf{S}^{1+1}$.

The generators of the one parameter subgroups of isometries, or Killing vector fields, are given as first-order differential operators in $(r, \phi)$ coordinates by:

$$
\left(\begin{array}{c}
X_{P_{1}} \\
X_{P_{2}} \\
X_{J}
\end{array}\right)=\left(\begin{array}{c}
C_{\kappa_{2}}(\phi) \partial_{r}-S_{\kappa_{2}}(\phi) / T_{\kappa_{1}}(r) \partial_{\phi} \\
\kappa_{2} S_{\kappa_{2}}(\phi) \partial_{r}+C_{\kappa_{2}}(\phi) / T_{\kappa_{1}}(r) \partial_{\phi} \\
\partial_{\phi}
\end{array}\right)
$$

and can be checked to close a Lie algebra isomorphic to $s o_{\kappa_{1}, \kappa_{2}}(3)$.

\subsection{Parallel ' 1 ' coordinates}

The coordinates are $(x, v)$. In this system the expressions are:

$$
\left(\begin{array}{c}
s^{0} \\
s^{1} \\
s^{2}
\end{array}\right)=\left(\begin{array}{c}
C_{\kappa_{1}}(x) C_{\kappa_{1} \kappa_{2}}(v) \\
S_{\kappa_{1}}(x) \\
C_{\kappa_{1}}(x) S_{\kappa_{1} \kappa_{2}}(v)
\end{array}\right), \quad d l^{2}=d x^{2}+\kappa_{2} C_{\kappa_{1}}^{2}(x) d v^{2} .
$$

On the standard Euclidean plane $\mathbf{E}^{2}$, with $\kappa_{1}=0, \kappa_{2}=1$ the coordinates $(x, v)$ are the usual cartesian coordinates and the metric reduces to $d l^{2}=d x^{2}+d v^{2}$. Likewise on 
the standard Minkowskian plane $\mathbf{M}^{1+1}\left(\kappa_{1}=0, \kappa_{2}=-1\right) x$ and $v$ are time and space Minkowskian coordinates with $d l^{2}=d x^{2}-d v^{2}$. The Killing vector fields associated to the basic one-parameter subgroups are:

$$
\left(\begin{array}{c}
X_{P_{1}} \\
X_{P_{2}} \\
X_{J}
\end{array}\right)=\left(\begin{array}{c}
C_{\kappa_{1} \kappa_{2}}(v) \partial_{x}+\kappa_{1} S_{\kappa_{1} \kappa_{2}}(v) T_{\kappa_{1}}(x) \partial_{v} \\
\partial_{v} \\
-\kappa_{2} S_{\kappa_{1} \kappa_{2}}(v) \partial_{x}+C_{\kappa_{1} \kappa_{2}}(v) T_{\kappa_{1}}(x) \partial_{v}
\end{array}\right) .
$$

\subsection{Parallel ' 2 ' coordinates}

The coordinates are $(u, y)$. In this case the analogous expressions are:

$$
\left(\begin{array}{c}
s^{0} \\
s^{1} \\
s^{2}
\end{array}\right)=\left(\begin{array}{c}
C_{\kappa_{1}}(u) C_{\kappa_{1} \kappa_{2}}(y) \\
S_{\kappa_{1}}(u) C_{\kappa_{1} \kappa_{2}}(y) \\
S_{\kappa_{1} \kappa_{2}}(y)
\end{array}\right), \quad d l^{2}=C_{\kappa_{1} \kappa_{2}}{ }^{2}(y) d u^{2}+\kappa_{2} d y^{2} .
$$

Note that the coordinate system $(u, y)$ does not coincide with $(x, v)$ in general; coincidence happens only when $\kappa_{1}=0$ and is a degeneracy of the flat spaces; for non zero values of $\kappa_{1}$ we have $u \neq x$ and $v \neq y$. On the standard sphere $\mathbf{S}^{2}(u, y)$ are the geographic longitude and colatitude coordinates. For the vector fields $X_{P_{1}}, X_{P_{2}}, X_{J}$ :

$$
\left(\begin{array}{c}
X_{P_{1}} \\
X_{P_{2}} \\
X_{J}
\end{array}\right)=\left(\begin{array}{c}
\partial_{u} \\
\kappa_{1} \kappa_{2} S_{\kappa_{1}}(u) T_{\kappa_{1} \kappa_{2}}(y) \partial_{u}+C_{\kappa_{1}}(u) \partial_{y} \\
-\kappa_{2} C_{\kappa_{1}}(u) T_{\kappa_{1} \kappa_{2}}(y) \partial_{u}+S_{\kappa_{1}}(u) \partial_{y}
\end{array}\right)
$$

which can be considered as the general version, valid for all CK spaces, of the well known Euclidean expressions $\partial_{u}, \partial_{y}, u \partial_{y}-y \partial_{u}$ for $X_{P_{1}}, X_{P_{2}}, X_{J}$.

\subsection{Equiparabolic '01' coordinates}

The coordinates, called here $\left(a_{+}, a_{-}\right)$, are defined as:

$$
a_{+}=\frac{1}{2}(r+x), \quad a_{-}=\frac{1}{2}(r-x) .
$$

and the point of the ambient CK space with coordinates $\left(a_{+}, a_{-}\right)$is given by

$$
\left(\begin{array}{c}
s^{0} \\
s^{1} \\
s^{2}
\end{array}\right)=\left(\begin{array}{c}
C_{\kappa_{1}}\left(a_{+}+a_{-}\right) \\
S_{\kappa_{1}}\left(a_{+}-a_{-}\right) \\
\sqrt{\frac{S_{\kappa_{1}}\left(2 a_{+}\right) S_{\kappa_{1}}\left(2 a_{-}\right)}{\kappa_{2}}}
\end{array}\right)=\left(\begin{array}{c}
C_{\kappa_{1}}(r) \\
S_{\kappa_{1}}(x) \\
\sqrt{\frac{S_{\kappa_{1}}(r+x) S_{\kappa_{1}}(r-x)}{\kappa_{2}}}
\end{array}\right),
$$

with metric:

$$
d l^{2}=\left(S_{\kappa_{1}}\left(2 a_{+}\right)+S_{\kappa_{1}}\left(2 a_{-}\right)\right)\left\{\frac{d a_{+}{ }^{2}}{S_{\kappa_{1}}\left(2 a_{+}\right)}+\frac{d a_{-}{ }^{2}}{S_{\kappa_{1}}\left(2 a_{-}\right)}\right\} .
$$

In spite of the presence of the constant $\kappa_{2}$ inside a square root in (3.6), due to the triangular inequality for lengths when $\kappa_{2}>0$ and to its (reversed) Minkowskian version when $\kappa_{2}<0$, the sign of $S_{\kappa_{1}}\left(2 a_{+}\right) S_{\kappa_{1}}\left(2 a_{-}\right)$turns out to be always the same as of $\kappa_{2}$, hence the three ambient space coordinates $s^{0}, s^{1}, s^{2}$ are always real. The prefactor in the line element can be written also in the form

$$
S_{\kappa_{1}}\left(2 a_{+}\right)+S_{\kappa_{1}}\left(2 a_{-}\right)=2 S_{\kappa_{1}}\left(a_{+}+a_{-}\right) C_{\kappa_{1}}\left(a_{+}-a_{-}\right)=2 S_{\kappa_{1}}(r) C_{\kappa_{1}}(x) .
$$


Also note that the actual orthogonal coordinates are $\left(a_{+}, a_{-}\right)$and, although the position of the generic point in the ambient space has been given in terms of $(r, x)$ in $(3.6)$, the coordinate system $(r, x)$ is not orthogonal. The same comment applies to the following equiparabolic systems.

Equiparabolic '01' coordinates are well defined and meaningful for any $S_{\left[\kappa_{1}\right] \kappa_{2}}^{2}$. In the standard Euclidean case $\mathbf{E}^{2}$ they reduce to the well known parabolic coordinates:

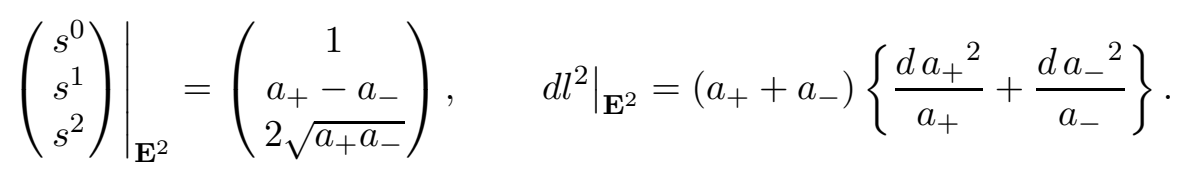

The "parabolic" name is thus justified as these coordinates involve sum and difference of distances to a focus and to a (focal) line. Here focus and focal line turn out to be incident and the prefix "equi" refers precisely to this incidence. In the general curved $S_{\left[\kappa_{1} \kappa_{2}\right.}^{2}$ there are "parabolic" type coordinates with nonzero distance between the focal point and the "focal line", a distance which thus plays a similar role to the interfocal distance for elliptic coordinates. Again we see that the Euclidean situation displays a zero curvature degeneracy.

The basic Killing vector fields associated to the three generators are:

$$
\begin{aligned}
X_{P_{1}} & =\frac{1}{S_{\kappa_{1}}\left(2 a_{+}\right)+S_{\kappa_{1}}\left(2 a_{-}\right)}\left(C_{\kappa_{1}}\left(2 a_{-}\right) S_{\kappa_{1}}\left(2 a_{+}\right) \partial_{a_{+}}-C_{\kappa_{1}}\left(2 a_{+}\right) S_{\kappa_{1}}\left(2 a_{-}\right) \partial_{a_{-}}\right), \\
X_{P_{2}} & =\frac{\sqrt{\kappa_{2}} \sqrt{S_{\kappa_{1}}\left(2 a_{+}\right) S_{\kappa_{1}}\left(2 a_{-}\right)}}{S_{\kappa_{1}}\left(2 a_{+}\right)+S_{\kappa_{1}}\left(2 a_{-}\right)} C_{\kappa_{1}}\left(a_{+}-a_{-}\right)\left(\partial_{a_{+}}+\partial_{a_{-}}\right) \\
X_{J} & =\frac{\sqrt{\kappa_{2}} \sqrt{S_{\kappa_{1}}\left(2 a_{+}\right) S_{\kappa_{1}}\left(2 a_{-}\right)}}{S_{\kappa_{1}}\left(2 a_{+}\right)+S_{\kappa_{1}}\left(2 a_{-}\right)} S_{\kappa_{1}}\left(a_{+}+a_{-}\right)\left(-\partial_{a_{+}}+\partial_{a_{-}}\right)
\end{aligned}
$$

\subsection{Equiparabolic '20' coordinates}

These coordinates, denoted $\left(b_{+}, b_{-}\right)$, are given by

$$
b_{+}=\frac{1}{2}\left(r+\sqrt{\kappa_{2}} y\right), \quad b_{-}=\frac{1}{2}\left(r-\sqrt{\kappa_{2}} y\right)
$$

and the point of the ambient CK space with coordinates $\left(b_{+}, b_{-}\right)$is given by

$$
\left(\begin{array}{c}
s^{0} \\
s^{1} \\
s^{2}
\end{array}\right)=\left(\begin{array}{c}
C_{\kappa_{1}}\left(b_{+}+b_{-}\right) \\
\sqrt{S_{\kappa_{1}}\left(2 b_{+}\right) S_{\kappa_{1}}\left(2 b_{-}\right)} \\
\frac{1}{\sqrt{\kappa_{2}}} S_{\kappa_{1}}\left(b_{+}-b_{-}\right)
\end{array}\right)=\left(\begin{array}{c}
C_{\kappa_{1}}(r) \\
\sqrt{S_{\kappa_{1}}\left(r+\sqrt{\kappa_{2}} y\right) S_{\kappa_{1}}\left(r-\sqrt{\kappa_{2}} y\right)} \\
S_{\kappa_{1} \kappa_{2}}(y)
\end{array}\right),
$$

for which the metric tensor follows from the line element:

$$
d l^{2}=\left(S_{\kappa_{1}}\left(2 b_{+}\right)+S_{\kappa_{1}}\left(2 b_{-}\right)\right)\left\{\frac{d b_{+}{ }^{2}}{S_{\kappa_{1}}\left(2 b_{+}\right)}+\frac{d b_{-}{ }^{2}}{S_{\kappa_{1}}\left(2 b_{-}\right)}\right\}
$$

the prefactor can be rewritten as:

$$
S_{\kappa_{1}}\left(2 b_{+}\right)+S_{\kappa_{1}}\left(2 b_{-}\right)=2 S_{\kappa_{1}}\left(b_{+}+b_{-}\right) C_{\kappa_{1}}\left(b_{+}-b_{-}\right)=2 S_{\kappa_{1}}(r) C_{\kappa_{1} \kappa_{2}}(y) .
$$


In the particular standard Euclidean case $\mathbf{E}^{2}$ the previous expressions reduce to the parabolic coordinates with axis along the line $l_{2}$ :

$$
\left.\left(\begin{array}{c}
s^{0} \\
s^{1} \\
s^{2}
\end{array}\right)\right|_{\mathbf{E}^{2}}=\left(\begin{array}{c}
1 \\
2 \sqrt{b_{+} b_{-}} \\
b_{+}-b_{-}
\end{array}\right),\left.\quad d l^{2}\right|_{\mathbf{E}^{2}}=\left(b_{+}+b_{-}\right)\left\{\frac{d b_{+}{ }^{2}}{b_{+}}+\frac{d b_{-}{ }^{2}}{b_{-}}\right\} .
$$

The basic Killing vector fields associated to the three generators are:

$$
\begin{aligned}
X_{P_{1}} & =\frac{\sqrt{S_{\kappa_{1}}\left(2 b_{+}\right) S_{\kappa_{1}}\left(2 b_{-}\right)}}{S_{\kappa_{1}}\left(2 b_{+}\right)+S_{\kappa_{1}}\left(2 b_{-}\right)} C_{\kappa_{1}}\left(b_{+}-b_{-}\right)\left(\partial_{b_{+}}+\partial_{b_{-}}\right) \\
X_{P_{2}} & =\frac{\sqrt{\kappa_{2}}}{S_{\kappa_{1}}\left(2 b_{+}\right)+S_{\kappa_{1}}\left(2 b_{-}\right)}\left(C_{\kappa_{1}}\left(2 b_{-}\right) S_{\kappa_{1}}\left(2 b_{+}\right) \partial_{b_{+}}-C_{\kappa_{1}}\left(2 b_{+}\right) S_{\kappa_{1}}\left(2 b_{-}\right) \partial_{b_{-}}\right), \\
X_{J} & =\frac{\sqrt{\kappa_{2}} \sqrt{S_{\kappa_{1}}\left(2 b_{+}\right) S_{\kappa_{1}}\left(2 b_{-}\right)}}{S_{\kappa_{1}}\left(2 b_{+}\right)+S_{\kappa_{1}}\left(2 b_{-}\right)} S_{\kappa_{1}}\left(b_{+}+b_{-}\right)\left(\partial_{b_{+}}-\partial_{b_{-}}\right)
\end{aligned}
$$

\subsection{Equiparabolic ' 12 ' coordinates}

Finally these coordinates, denoted $\left(z_{+}, z_{-}\right)$, are defined as:

$$
z_{+}=\frac{1}{2}\left(\sqrt{\kappa_{2}} y+x\right), \quad z_{-}=\frac{1}{2}\left(\sqrt{\kappa_{2}} y-x\right) .
$$

The point on the ambient space is:

$$
\left(\begin{array}{c}
s^{0} \\
s^{1} \\
s^{2}
\end{array}\right)=\left(\begin{array}{c}
\sqrt{C_{\kappa_{1}}\left(2 z_{+}\right) C_{\kappa_{1}}\left(2 z_{-}\right)} \\
S_{\kappa_{1}}\left(z_{+}-z_{-}\right) \\
\frac{1}{\sqrt{\kappa_{2}}} S_{\kappa_{1}}\left(z_{+}+z_{-}\right)
\end{array}\right)=\left(\begin{array}{c}
\sqrt{C_{\kappa_{1}}\left(\sqrt{\kappa_{2}} y+x\right) C_{\kappa_{1}}\left(\sqrt{\kappa_{2}} y-x\right)} \\
S_{\kappa_{1}}(x) \\
S_{\kappa_{1} \kappa_{2}}(y)
\end{array}\right)
$$

and the metric tensor follows from the line element:

$$
d l^{2}=\left(C_{\kappa_{1}}\left(2 z_{+}\right)+C_{\kappa_{1}}\left(2 z_{-}\right)\right)\left\{\frac{d z_{+}{ }^{2}}{C_{\kappa_{1}}\left(2 z_{+}\right)}+\frac{d z_{-}{ }^{2}}{C_{\kappa_{1}}\left(2 z_{-}\right)}\right\}
$$

where the prefactor $C_{\kappa_{1}}\left(2 z_{+}\right)+C_{\kappa_{1}}\left(2 z_{-}\right)$can be rewritten as $2 C_{\kappa_{1}}(x) C_{\kappa_{1} \kappa_{2}}(y)$. In the standard Euclidean $\mathbf{E}^{2}$ the coordinates $\left(z_{+}, z_{-}\right)$coincide with the cartesian ones relative to a system the axis of which has been rotated by half a quadrant:

$$
\left.\left(\begin{array}{c}
s^{0} \\
s^{1} \\
s^{2}
\end{array}\right)\right|_{\mathbf{E}^{2}}=\left(\begin{array}{c}
1 \\
z_{+}-z_{-} \\
z_{+}+z_{-}
\end{array}\right)
$$

but again this identification with some parallel coordinates is a degeneracy of the flat case; as soon as $\kappa_{1} \neq 0$, these coordinates are essentially new and only in the flat spaces bear some simple relation to a rotated cartesian type system. The name equiparabolic '12' for these coordinates is due to their relation, through $T$-symmetry to be mentioned later, to the equiparabolic ' 20 ' and ' 01 ' coordinates.

To conclude, we give the Killing vector fields associated to the three generators:

$$
X_{P_{1}}=\frac{\sqrt{C_{\kappa_{1}}\left(2 z_{+}\right) C_{\kappa_{1}}\left(2 z_{-}\right)}}{C_{\kappa_{1}}\left(2 z_{+}\right)+C_{\kappa_{1}}\left(2 z_{-}\right)} C_{\kappa_{1}}\left(z_{+}+z_{-}\right)\left(\partial_{z_{+}}-\partial_{z_{-}}\right)
$$




$$
\begin{aligned}
X_{P_{2}} & =\frac{\sqrt{\kappa_{2}} \sqrt{C_{\kappa_{1}}\left(2 z_{+}\right) C_{\kappa_{1}}\left(2 z_{-}\right)}}{C_{\kappa_{1}}\left(2 z_{+}\right)+C_{\kappa_{1}}\left(2 z_{-}\right)} C_{\kappa_{1}}\left(z_{+}-z_{-}\right)\left(\partial_{z_{+}}+\partial_{z_{-}}\right), \\
X_{J} & =\frac{\sqrt{\kappa_{2}}}{C_{\kappa_{1}}\left(2 z_{+}\right)+C_{\kappa_{1}}\left(2 z_{-}\right)}\left(-S_{\kappa_{1}}\left(2 z_{-}\right) C_{\kappa_{1}}\left(2 z_{+}\right) \partial_{z_{+}}+S_{\kappa_{1}}\left(2 z_{+}\right) C_{\kappa_{1}}\left(2 z_{-}\right) \partial_{z_{-}}\right) .
\end{aligned}
$$

\subsection{On the relation between this approach and the coordinate systems separating the Laplacian}

In each CK space (hence in all the spaces in Table 1) the orthogonal coordinate systems separating the Laplace-Beltrami (LB) equation can be understood as either generic or particular/limiting cases of the general elliptic coordinates in each space. A complete discussion on this viewpoint on the classification will be given elsewhere [27]. These systems are well known in the $2 \mathrm{~d}$ sphere $\mathbf{S}^{2}$, Euclidean plane $\mathbf{E}^{2}$ and hyperbolic plane $\mathbf{H}^{2}$ [19]; for $\mathbf{M}^{1+1}$ see [12] and [16]. Now we relate these classifications to the coordinate systems we have discussed. To ease this comparison, in this subsection the names we propose are written in quotes, and the names found in these references are in plain text. Up to motions of the isometry group for each space, coordinate systems separating the LB operator belong to one of the several classes:

For the sphere $\mathbf{S}^{2}$, there are only two classes: polar and elliptic. The later is generic and depend on a modulus (the focal distance $2 f$ ); polar is the limiting instance $2 f \rightarrow 0$.

In the Euclidean plane $\mathbf{E}^{2}$ there are four classes: polar, cartesian, parabolic and elliptic. The later is generic and depends on a modulus (the focal distance $2 f$ ); polar class is the limiting case $2 f \rightarrow 0$ and cartesian and parabolic are two different limiting cases $2 f \rightarrow \infty$.

For the hyperbolic plane $\mathbf{H}^{2}$ there are nine classes. Three of them (elliptic, hyperbolic and semi-hyperbolic) are generic and depend on a modulus, while the remaining six (polar, equidistant, elliptic parabolic, hyperbolic parabolic, osculating parabolic and horocyclic) are limiting or particular cases.

In the Minkowski space $\mathbf{M}^{1+1}$ there are generic class, which depend on a modulus (this can be interpreted also as the suitable focal separation for the generic 'elliptic' system $[27])$ and the remaining systems are limiting or particular cases.

It is interesting to mention here how the six coordinate systems we are discussing in this paper are placed amongst these more general ones separating the LB equation.

On the sphere $\mathbf{S}^{2}$ the three 'polar', 'parallel 1' and 'parallel 2' coordinates are equivalent (they are related by triality which in $\mathbf{S}^{2}$ is a proper rotation belonging to the isometry group); they fall in the polar class. Likewise, the three 'equiparabolic 01, 20 and 12' systems are equivalent and they are the very special self-complementary instance (with the focal distance equal a quadrant) of elliptic class on the sphere.

On the Euclidean plane $\mathbf{E}^{2}$ our 'polar' is polar class, our 'parallel 1' and 'parallel 2' are equivalent and are in the cartesian class, our 'equiparabolic 01 and 20 ' are equivalent and belong to the parabolic class, and finally our 'equiparabolic 12' is in the cartesian class, though with rotated axis by an angle of half a quadrant relative to the 'parallel 1' and 'parallel 2' cartesian ones. Thus our systems correspond to the polar, cartesian and parabolic class.

On the hyperbolic plane $\mathbf{H}^{2}$, our 'polar' is in the polar or pseudospheric class, our 'parallel 1 and 2' are equivalent and are in the equidistant class, our 'equiparabolic 01 
and $20^{\prime}$ are equivalent and very special instances (with vanishing value for the modulus) of the semi-hyperbolic class, and finally our 'equiparabolic 12' is the very special selfcomplementary instance of hyperbolic class where the focal angle equals to a quadrant; hence on $\mathbf{H}^{2}$ our six sytems fall into four classes, two of them without modulus and two special instances, with specific values for the corresponding moduli, of the semi-hyperbolic and hyperbolic class.

\section{Separable potentials}

Starting from the Lagrangian

$$
L=\frac{1}{2} g_{\mu \nu}\left(q^{1}, q^{2}\right) \dot{q^{\mu}} \dot{q}^{\nu}-V\left(q^{1}, q^{2}\right),
$$

we may define the Legendre transformation by $p_{\mu}=\partial L / \partial \dot{q}^{\mu}$ and introduce the Hamiltonian:

$$
H=\frac{1}{2} g^{\mu \nu}\left(q^{1}, q^{2}\right) p_{\mu} p_{\nu}+V\left(q^{1}, q^{2}\right),
$$

where as always the constants $\kappa_{1}$ and $\kappa_{2}$ are considered as parameters. This transition is only possible for the non degenerate CK spaces for which $\kappa_{2} \neq 0$. The "energy" is a constant of motion which is quadratic in the momenta. Are there other additional constants of motion also quadratic in the momenta?. To discuss this it is better to look first for the possible constants of motion which are linear in the momenta. This happens when the Lagrangian has a Killing vector field as an exact Noether symmetry. In particular the Noether constant associated to the invariance under the one-parameter subgroup generated by $X_{Y}$ can be expressed as the image $\Theta_{L}\left(X_{Y}^{T}\right)$, under the Cartan semibasic one-form $\Theta_{L}:=\partial L / \partial q^{\mu} d q^{\mu}$ of the natural lift $X_{Y}^{T}$ to the tangent bundle (phase space) of the vector field $X_{Y}$ [18]. By making some convenient abuse of language we call $P_{1}, P_{2}, J$ the Noether constants associated to the invariance under the three one-parameter subgroups $\exp \left(\alpha P_{1}\right)$, $\exp \left(\beta P_{2}\right)$ or $\exp (\gamma J)$. Directly we find the expressions for these possible constants in terms either of the momenta or of the coordinate velocities:

- In Polar coordinates

$$
\begin{gathered}
p_{r}=\dot{r}, \quad p_{\phi}=\kappa_{2} S_{\kappa_{1}}^{2}(r) \dot{\phi} \\
\left(\begin{array}{c}
P_{1} \\
P_{2} \\
J
\end{array}\right)=\left(\begin{array}{c}
C_{\kappa_{2}}(\phi) p_{r}-S_{\kappa_{2}}(\phi) / T_{\kappa_{1}}(r) p_{\phi} \\
\kappa_{2} S_{\kappa_{2}}(\phi) p_{r}+C_{\kappa_{2}}(\phi) / T_{\kappa_{1}}(r) p_{\phi} \\
p_{\phi}
\end{array}\right)=\left(\begin{array}{c}
C_{\kappa_{2}}(\phi) \dot{r}-\kappa_{2} C_{\kappa_{1}}(r) S_{\kappa_{1}}(r) S_{\kappa_{2}}(\phi) \dot{\phi} \\
\kappa_{2} S_{\kappa_{2}}(\phi) \dot{r}+\kappa_{2} C_{\kappa_{1}}(r) S_{\kappa_{1}}(r) C_{\kappa_{2}}(\phi) \dot{\phi} \\
\kappa_{2} S_{\kappa_{1}}{ }^{2}(r) \dot{\phi}
\end{array}\right) .
\end{gathered}
$$

- In Parallel ' 1 ' coordinates

$$
\begin{gathered}
p_{x}=\dot{x}, \quad p_{v}=\kappa_{2} C_{\kappa_{1}}^{2}(x) \dot{v} \\
\left(\begin{array}{c}
P_{1} \\
P_{2} \\
J
\end{array}\right)=\left(\begin{array}{c}
C_{\kappa_{1} \kappa_{2}}(v) p_{x}+\kappa_{1} S_{\kappa_{1} \kappa_{2}}(v) T_{\kappa_{1}}(x) p_{v} \\
p_{v} \\
-\kappa_{2} S_{\kappa_{1} \kappa_{2}}(v) p_{x}+C_{\kappa_{1} \kappa_{2}}(v) T_{\kappa_{1}}(x) p_{v}
\end{array}\right)=\left(\begin{array}{c}
C_{\kappa_{1} \kappa_{2}}(v) \dot{x}+\kappa_{1} \kappa_{2} S_{\kappa_{1} \kappa_{2}}(v) S_{\kappa_{1}}(x) C_{\kappa_{1}}(x) \dot{v} \\
\kappa_{2} C_{\kappa_{1}}(x) \dot{v} \\
-\kappa_{2} S_{\kappa_{1} \kappa_{2}}(v) \dot{x}+\kappa_{2} C_{\kappa_{1} \kappa_{2}}(v) S_{\kappa_{1}}(x) C_{\kappa_{1}}(x) \dot{v}
\end{array}\right) .
\end{gathered}
$$

- In Parallel '2' coordinates

$$
p_{u}=C_{\kappa_{1} \kappa_{2}}^{2}(y) \dot{u}, \quad p_{y}=\kappa_{2} \dot{y}
$$




$$
\left(\begin{array}{c}
P_{1} \\
P_{2} \\
J
\end{array}\right)=\left(\begin{array}{c}
p_{u} \\
\kappa_{1} \kappa_{2} S_{\kappa_{1}}(u) T_{\kappa_{1} \kappa_{2}}(y) p_{u}+C_{\kappa_{1}}(u) p_{y} \\
-\kappa_{2} C_{\kappa_{1}}(u) T_{\kappa_{1} \kappa_{2}}(y) p_{u}+S_{\kappa_{1}}(u) p_{y}
\end{array}\right)=\left(\begin{array}{c}
C_{\kappa_{1} \kappa_{2}}^{2}(y) \dot{u} \\
\kappa_{1} \kappa_{2} S_{\kappa_{1}}(u) S_{\kappa_{1} \kappa_{2}}(y) C_{\kappa_{1} \kappa_{2}}(y) \dot{u}+\kappa_{2} C_{\kappa_{1}}(u) \dot{y} \\
-\kappa_{2} C_{\kappa_{1}}(u) S_{\kappa_{1} \kappa_{2}}(y) C_{\kappa_{1} \kappa_{2}}(y) \dot{u}+\kappa_{2} S_{\kappa_{1}}(u) \dot{y}
\end{array}\right) .
$$

In the next three 'equiparabolic' systems we give the expressions of the canonical momenta $p_{a_{+}}$etc. and of the Noether momenta $P_{1}$ etc. in terms of coordinate velocities. Noether momenta in terms of canonical momenta are obtained by letting $\Theta_{L}$ act on the Killing vector fields $X_{P_{1}}$ etc., which amounts to replace partial derivative operators by the corresponding canonical momenta $\partial_{a_{+}} \rightarrow p_{a_{+}}$etc.

- In Equiparabolic '01' coordinates

$$
\begin{aligned}
p_{a_{+}} & =\left(S_{\kappa_{1}}\left(2 a_{+}\right)+S_{\kappa_{1}}\left(2 a_{-}\right)\right) \frac{\dot{a_{+}}}{S_{\kappa_{1}}\left(2 a_{+}\right)}, \quad p_{a_{-}}=\left(S_{\kappa_{1}}\left(2 a_{+}\right)+S_{\kappa_{1}}\left(2 a_{-}\right)\right) \frac{\dot{a_{-}}}{S_{\kappa_{1}}\left(2 a_{-}\right)}, \\
P_{1} & =C_{\kappa_{1}}\left(2 a_{-}\right) a_{+}-C_{\kappa_{1}}\left(2 a_{+}\right) \dot{a_{-}}, \\
P_{2} & =\sqrt{\kappa_{2}} \sqrt{S_{\kappa_{1}}\left(2 a_{+}\right) S_{\kappa_{1}}\left(2 a_{-}\right)} C_{\kappa_{1}}\left(a_{+}-a_{-}\right)\left(\frac{\dot{a_{+}}}{S_{\kappa_{1}}\left(2 a_{+}\right)}+\frac{\dot{a_{-}}}{S_{\kappa_{1}}\left(2 a_{-}\right)}\right), \\
J & =\sqrt{\kappa_{2}} \sqrt{S_{\kappa_{1}}\left(2 a_{+}\right) S_{\kappa_{1}}\left(2 a_{-}\right)} S_{\kappa_{1}}\left(a_{+}+a_{-}\right)\left(-\frac{\dot{a_{+}}}{S_{\kappa_{1}}\left(2 a_{+}\right)}+\frac{\dot{a_{-}}}{S_{\kappa_{1}}\left(2 a_{-}\right)}\right),
\end{aligned}
$$

- In Equiparabolic '20' coordinates

$$
\begin{aligned}
p_{b_{+}} & =\left(S_{\kappa_{1}}\left(2 b_{+}\right)+S_{\kappa_{1}}\left(2 b_{-}\right)\right) \frac{\dot{b_{+}}}{S_{\kappa_{1}}\left(2 b_{+}\right)}, \quad p_{b_{-}}=\left(S_{\kappa_{1}}\left(2 b_{+}\right)+S_{\kappa_{1}}\left(2 b_{-}\right)\right) \frac{\dot{b_{-}}}{S_{\kappa_{1}}\left(2 b_{-}\right)} . \\
P_{1} & =\sqrt{S_{\kappa_{1}}\left(2 b_{+}\right) S_{\kappa_{1}}\left(2 b_{-}\right)} C_{\kappa_{1}}\left(b_{+}-b_{-}\right)\left(\frac{\dot{b_{+}}}{S_{\kappa_{1}}\left(2 b_{+}\right)}+\frac{\dot{b_{-}}}{S_{\kappa_{1}}\left(2 b_{-}\right)}\right) \\
P_{2} & =\sqrt{\kappa_{2}}\left(C_{\kappa_{1}}\left(2 b_{-}\right) \dot{b_{+}}-C_{\kappa_{1}}\left(2 b_{+}\right) \dot{b_{-}}\right) \\
J & =\sqrt{\kappa_{2}} \sqrt{S_{\kappa_{1}}\left(2 b_{+}\right) S_{\kappa_{1}}\left(2 b_{-}\right)} S_{\kappa_{1}}\left(b_{+}+b_{-}\right)\left(\frac{\dot{b_{+}}}{S_{\kappa_{1}}\left(2 b_{+}\right)}-\frac{\dot{b_{-}}}{S_{\kappa_{1}}\left(2 b_{-}\right)}\right) .
\end{aligned}
$$

- In Equiparabolic '12' coordinates

$$
\begin{aligned}
p_{z_{+}}= & \left(C_{\kappa_{1}}\left(2 z_{+}\right)+C_{\kappa_{1}}\left(2 z_{-}\right)\right) \frac{\dot{z_{+}}}{C_{\kappa_{1}}\left(2 z_{+}\right)}, \quad p_{z_{-}}=\left(C_{\kappa_{1}}\left(2 z_{+}\right)+C_{\kappa_{1}}\left(2 z_{-}\right)\right) \frac{\dot{z_{-}}}{C_{\kappa_{1}}\left(2 z_{-}\right)}, \\
P_{1} & =\sqrt{C_{\kappa_{1}}\left(2 z_{+}\right) C_{\kappa_{1}}\left(2 z_{-}\right)} C_{\kappa_{1}}\left(z_{+}+z_{-}\right)\left(\frac{\dot{z_{+}}}{C_{\kappa_{1}}\left(2 z_{+}\right)}-\frac{\dot{z_{-}}}{C_{\kappa_{1}}\left(2 z_{-}\right)}\right), \\
P_{2} & =\sqrt{\kappa_{2}} \sqrt{C_{\kappa_{1}}\left(2 z_{+}\right) C_{\kappa_{1}}\left(2 z_{-}\right)} C_{\kappa_{1}}\left(z_{+}-z_{-}\right)\left(\frac{\dot{z_{+}}}{C_{\kappa_{1}}\left(2 z_{+}\right)}+\frac{\dot{z_{-}}}{C_{\kappa_{1}}\left(2 z_{-}\right)}\right), \\
J & =\sqrt{\kappa_{2}}\left(-S_{\kappa_{1}}\left(2 z_{-}\right) \dot{z_{+}}+S_{\kappa_{1}}\left(2 z_{+}\right) \dot{z_{-}}\right) .
\end{aligned}
$$

The Noether momentum $P_{1}$ (resp. $P_{2}, J$ ) is itself a constant of motion if the potential is invariant under the one-parameter subgroup generated by $P_{1}\left(\operatorname{resp} . P_{2}, J\right)$, i.e., when $X_{P_{1}} V=0$ (resp. $X_{P_{2}} V=0, X_{J} V=0$ ). Although this condition is meaningful in any coordinates, it is simpler in the parallel '2' (resp. parallel ' 1 ' and polar), where it means that $V$ has no dependence on $u$ (resp. on $v, \phi$ ) and depends only on $y$ (resp. only on $x, r$ ). 
Now we focus attention on potentials $V\left(q^{1}, q^{2}\right)$ which are endowed with constants of motion quadratic in the velocities. The quadratic integral $I$ that is given by

$$
2 I=k_{11}\left(q^{1}, q^{2}\right)\left(\dot{q}^{1}\right)^{2}+2 k_{12}\left(q^{1}, q^{2}\right) \dot{q}^{1} \dot{q}^{2}+k_{22}\left(q^{1}, q^{2}\right)\left(\dot{q}^{2}\right)^{2}+2 W\left(q^{1}, q^{2}\right),
$$

can be, equivalently, expressed as a function quadratic in the Noether momenta

$$
2 I=a_{0} J^{2}+a_{1} P_{2}^{2}+a_{2} P_{1}^{2}+2 a_{12} P_{1} P_{2}+2 a_{20} J P_{1}+2 a_{01} J P_{2}+2 W\left(q^{1}, q^{2}\right),
$$

where $k_{11}, k_{12}=k_{21}, k_{22}$ and $W$ depend on $\left(q^{1}, q^{2}\right)$ and $a_{0}, a_{1}, a_{2}, a_{12}, a_{20}, a_{01}$ are numerical constants. Any potential has always such a constant, the $\left(\kappa_{1}, \kappa_{2}\right)$-energy, the quadratic part of which is the Casimir of the isometry algebra, but only very specific potentials allow additional quadratic constants of motion of $I$ type.

Why are these two forms equivalent to each other? If we enforce $\dot{I}=0$ in the first form, use the Euler-Lagrange equations and separate coefficients, we get two sets of equations. The first set involves only $k_{11}, k_{12}=k_{21}, k_{22}$ and states the symmetric tensor $k_{\mu \nu}$ is a special conformal Killing tensor $[1,2,17]$. Once the general solution for these equations has been found, this leads to:

$$
k_{11}\left(\dot{q}^{1}\right)^{2}+2 k_{12} \dot{q}^{1} \dot{q}^{2}+k_{22}\left(\dot{q}^{2}\right)^{2}=a_{0} J^{2}+a_{1} P_{2}^{2}+a_{2} P_{1}^{2}+2 a_{12} P_{1} P_{2}+2 a_{20} J P_{1}+2 a_{01} J P_{2},
$$

with $a_{0}, a_{1}, \ldots$ independent of coordinates. The second set determines $\partial_{1} W, \partial_{2} W$ in terms of $V, k_{\mu \nu}$. The compatibility condition for this set of equations has the following form for orthogonal coordinate systems:

$$
\partial_{2}\left(\frac{k_{11}}{g_{11}} \partial_{1} V+\frac{k_{12}}{g_{22}} \partial_{2} V\right)=\partial_{1}\left(\frac{k_{21}}{g_{11}} \partial_{1} V+\frac{k_{22}}{g_{22}} \partial_{2} V\right)
$$

Thus in order to have $I$ as a constant of motion, the potential must satisfy this differential equation which depends linearly on the numerical constants $a_{0}, a_{1}, a_{2}, a_{12}, a_{20}, a_{01}$. Explicit checkings require the knowledge of $k_{11}, k_{12}=k_{21}, k_{22}$, but these are most conveniently expressed through (4.5) in terms of the Noether momenta the expressions of which have been given for the coordinate systems under consideration in the previous page.

For completeness we first state a trivial result:

Theorem 1. Any potential allows for a constant of motion of type $I$, the "energy" $I_{E}$, whose quadratic part is of the form $\kappa_{2} P_{1}^{2}+P_{2}^{2}+\kappa_{1} J_{2}$ :

$$
2 I_{E}=g_{\mu \nu}\left(q^{1}, q^{2}\right) \dot{q}^{\mu} \dot{q}^{\nu}+2 V\left(q^{1}, q^{2}\right)=\frac{1}{\kappa_{2}}\left(\kappa_{1} J^{2}+\kappa_{2} P_{1}^{2}+P_{2}^{2}\right)+2 V\left(q^{1}, q^{2}\right) .
$$

A remark is in order here. In the standard Euclidean case the energy has the well known expression $\frac{1}{2}\left\{P_{1}^{2}+P_{2}^{2}\right\}+V$, which has a contribution from the two Noether translational or linear momenta, $P_{1}$ and $P_{2}$, but none from the angular momentum $J$. This property is a degeneracy of the flat case and, as soon as the curvature of the configuration space is nonzero, the angular momentum has a quadratic contribution to the energy. In the theorem "energy" is in quotes because, when the configuration space is locally Minkowskian, the physical meaning of this constant is rather different to the usual energy. This constant should be interpreted as the physical energy of a non relativistic particle moving in a configuration space with nonzero curvature $\kappa_{1}$ only when $\kappa_{2}>0$. 
The possibility of extra constants of motion is described in the following statements, where non-degenerate $S_{\left[\kappa_{1}\right] \kappa_{2}}^{2}$ means a CK $2 \mathrm{~d}$ space with any curvature (either positive, zero or negative) and a non degenerate metric (thus $\kappa_{2} \neq 0$ ).

Theorem 2. For any non-degenerate $S_{\left[\kappa_{1}\right] \kappa_{2}}^{2}$, potentials allowing for an extra constant of motion $I_{J^{2}}$ the quadratic part of which is of the form $a_{0} J^{2}$ are precisely those with a dependency in the polar coordinate system given by:

$$
V(r, \phi)=\frac{1}{S_{\kappa_{1}}^{2}(r)}\{\mathcal{A}(r)+\mathcal{B}(\phi)\},
$$

and the constant of motion is:

$$
2 I_{J^{2}}=J^{2}+2 \kappa_{2} \mathcal{B}(\phi) .
$$

Theorem 3. For any non-degenerate $S_{\left[\kappa_{1}\right] \kappa_{2}}^{2}$, potentials allowing for an extra constant of motion $I_{P_{2}^{2}}$ the quadratic part of which is of the form $a_{1} P_{2}^{2}$ are precisely those those with a dependency given in parallel ' 1 ' coordinates by:

$$
V(x, v)=\frac{1}{C_{\kappa_{1}}^{2}(x)}\{\mathcal{A}(x)+\mathcal{B}(v)\},
$$

and the constant of motion is:

$$
2 I_{P_{2}^{2}}=P_{2}^{2}+2 \kappa_{2} \mathcal{B}(v) .
$$

Theorem 4. For any non-degenerate $S_{\left[\kappa_{1}\right] \kappa_{2}}^{2}$, potentials allowing for an extra constant of motion $I_{P_{1}^{2}}$ the quadratic part of which is of the form $a_{2} P_{1}^{2}$ are precisely those with a dependency given in the parallel ' 2 ' coordinate system by:

$$
V(u, y)=\frac{1}{C_{\kappa_{1} \kappa_{2}}^{2}(y)}\{\mathcal{A}(y)+\mathcal{B}(u)\}
$$

and the constant of motion is:

$$
2 I_{P_{1}^{2}}=\kappa_{2} P_{1}^{2}+2 \kappa_{2} \mathcal{B}(u) .
$$

Theorem 5. For any non-degenerate $S_{\left[\kappa_{1}\right] \kappa_{2}}^{2}$, potentials allowing for an extra constant of motion the quadratic part of which is of the form $a_{12} P_{1} P_{2}$ are precisely those separable in the equiparabolic ' $1 \mathbf{2}$ ' coordinate system, this is those with a dependency:

$$
V\left(z_{+}, z_{-}\right)=\frac{1}{C_{\kappa_{1}}\left(2 z_{+}\right)+C_{\kappa_{1}}\left(2 z_{-}\right)}\left\{\mathcal{A}\left(z_{+}\right)+\mathcal{B}\left(z_{-}\right)\right\}=\frac{1}{2 C_{\kappa_{1}}(x) C_{\kappa_{1} \kappa_{2}}(y)}\left\{\mathcal{A}\left(z_{+}\right)+\mathcal{B}\left(z_{-}\right)\right\},
$$

and the constant of motion $I_{P_{1} P_{2}}$ is:

$$
I_{P_{1} P_{2}}=-\sqrt{\kappa_{2}} P_{1} P_{2}+\frac{\kappa_{2}}{C_{\kappa_{1}}\left(2 z_{+}\right)+C_{\kappa_{1}}\left(2 z_{-}\right)}\left\{C_{\kappa_{1}}\left(2 z_{+}\right) \mathcal{B}\left(z_{-}\right)-C_{\kappa_{1}}\left(2 z_{-}\right) \mathcal{A}\left(z_{+}\right)\right\} .
$$


Theorem 6. For any non-degenerate $S_{\left[\kappa_{1}\right] \kappa_{2}}^{2}$, potentials allowing for an extra constant of motion the quadratic part of which is of the form $a_{20} J P_{1}$ are precisely those with a dependency in equiparabolic ' 20 ' coordinates as :

$$
V\left(b_{+}, b_{-}\right)=\frac{1}{S_{\kappa_{1}}\left(2 b_{+}\right)+S_{\kappa_{1}}\left(2 b_{-}\right)}\left\{\mathcal{A}\left(b_{-}\right)+\mathcal{B}\left(b_{+}\right)\right\}=\frac{1}{2 S_{\kappa_{1}}(r) C_{\kappa_{1} \kappa_{2}}(y)}\left\{\mathcal{A}\left(b_{-}\right)+\mathcal{B}\left(b_{+}\right)\right\},
$$

and the constant of motion $I_{J P_{1}}$ is:

$$
I_{J P_{1}}=\sqrt{\kappa_{2}} J P_{1}+\frac{\kappa_{2}}{S_{\kappa_{1}}\left(2 b_{+}\right)+S_{\kappa_{1}}\left(2 b_{-}\right)}\left\{S_{\kappa_{1}}\left(2 b_{-}\right) \mathcal{B}\left(b_{+}\right)-S_{\kappa_{1}}\left(2 b_{+}\right) \mathcal{A}\left(b_{-}\right)\right\} .
$$

Theorem 7. For any non-degenerate $S_{\left[\kappa_{1}\right] \kappa_{2}}^{2}$, potentials allowing for an extra constant of motion the quadratic part of which is of the form $a_{01} J P_{2}$ are precisely those with a dependency given in equiparabolic ' 01 ' coordinates as:

$$
V\left(a_{+}, a_{-}\right)=\frac{1}{S_{\kappa_{1}}\left(2 a_{+}\right)+S_{\kappa_{1}}\left(2 a_{-}\right)}\left\{\mathcal{A}\left(a_{-}\right)+\mathcal{B}\left(a_{+}\right)\right\}=\frac{1}{2 S_{\kappa_{1}}(r) C_{\kappa_{1}}(x)}\left\{\mathcal{A}\left(a_{-}\right)+\mathcal{B}\left(a_{+}\right)\right\},
$$

and the constant of motion further to the energy is:

$$
I_{J P_{2}}=-J P_{2}+\frac{\kappa_{2}}{S_{\kappa_{1}}\left(2 a_{+}\right)+S_{\kappa_{1}}\left(2 a_{-}\right)}\left\{S_{\kappa_{1}}\left(2 a_{-}\right) \mathcal{B}\left(a_{+}\right)-S_{\kappa_{1}}\left(2 a_{+}\right) \mathcal{A}\left(a_{-}\right)\right\} .
$$

All the proofs reduce to routine computation and indeed follow from the (Stäckel) form of the metrics. While some of these results have been long known for particular spaces, the novel part presented in this paper concerns the joint treatment, displaying aspects which cannot be seen in each particular space and points clearly to the degeneracies specific of the flat spaces; therefore any study the philosophy of which is to start from the Euclidean situation meets unnecesary dificulties; the opposite approach, considering first the generic $\kappa_{1}, \kappa_{2}$ situation and only then specializing to each particular case, is likely to be much more illuminating.

\subsection{Hamilton-Jacobi separability}

The Hamilton-Jacobi equation associated to a potential $V\left(q^{1}, q^{2}\right)$ is:

$$
\frac{1}{2} g^{\mu \nu}\left(q^{1}, q^{2}\right) \frac{\partial S}{\partial q^{\mu}} \frac{\partial S}{\partial q^{\nu}}+V\left(q^{1}, q^{2}\right)=E .
$$

Now this equation admits separation of variables if the general solution can be expressed by means of separated solutions, having the form:

$$
S\left(q^{1}, q^{2}\right)=\left\{\mathcal{M}\left(q^{1}\right)+\mathcal{N}\left(q^{2}\right)\right\} .
$$

From general results we may re state the results in the previous section as:

Theorem 8. For any non degenerate $S_{\left[\kappa_{1}\right] \kappa_{2}}^{2}$, all the six coordinate systems described above allow separation of variables in the free Hamilton-Jacobi equation.

Theorem 9. For any non degenerate $S_{\left[\kappa_{1}\right] \kappa_{2}}^{2}$, the polar coordinate system allows separation of variables in the Hamilton-Jacobi equation whenever the potential has the form (4.8)

$\ldots$ and so on for the six coordinate systems. 


\section{$5 \quad$ T-symmetry}

When $\kappa_{1}$ and $\kappa_{2}$ are positive, the full group $O_{\kappa_{1}, \kappa_{2}}(3)$ contains a discrete finite subgroup $T_{\kappa_{1}, \kappa_{2}}$ isomorphic to the octahedral group $T$. Further to the trivial reflections in the three planes $s^{0}=0, s^{1}=0, s^{2}=0$, this group is generated by an 3 -fold rotation $\tau$ around the 'center' of the first octant and a reflection $\sigma$, acting in the ambient space as:

$$
\left(\begin{array}{c}
s^{0} \\
s^{1} \\
s^{2}
\end{array}\right) \stackrel{\tau}{\longrightarrow}\left(\begin{array}{c}
\sqrt{\kappa_{1}} s^{1} \\
\sqrt{\kappa_{2}} s^{2} \\
\left(1 / \sqrt{\kappa_{1} \kappa_{2}}\right) s^{0}
\end{array}\right), \quad\left(\begin{array}{c}
s^{0} \\
s^{1} \\
s^{2}
\end{array}\right) \stackrel{\sigma}{\rightarrow}\left(\begin{array}{c}
s^{0} \\
\sqrt{\kappa_{2}} s^{2} \\
\left(1 / \sqrt{\kappa_{2}}\right) s^{1}
\end{array}\right) .
$$

Assume for the moment that $\kappa_{1}>0, \kappa_{2}>0$. Then $\tau$ transforms cyclically between the coordinates in the three basic systems $(r, \phi),(u, y),(x, v)$ according to the rules (the $\sqrt{\kappa_{1}}, \sqrt{\kappa_{2}}$ factors ensure all quantities are transferred to have label $\kappa_{1}$, or equivalently, that all quantities are measured in the ordinary angular scale used in $\mathbf{S}^{2}$ for lengths):

$$
r \stackrel{\tau}{\longrightarrow} \widetilde{x} \stackrel{\tau}{\longrightarrow} \widetilde{\sqrt{\kappa_{2}} y} \stackrel{\tau}{\longrightarrow} r, \quad \sqrt{\frac{\kappa_{2}}{\kappa_{1}}} \phi \stackrel{\tau}{\longrightarrow} \widetilde{\sqrt{\kappa_{2}} v} \stackrel{\tau}{\longrightarrow} u \stackrel{\tau}{\longrightarrow} \sqrt{\frac{\kappa_{2}}{\kappa_{1}}} \phi,
$$

where, if $x$ is any variable with label $\kappa, \widetilde{x}$ denotes the complement of $x$ defined so that

$$
x=\frac{\pi / 2}{\sqrt{\kappa}}-\widetilde{x}, \quad \text { with } \quad C_{\kappa}(\widetilde{x})=\sqrt{\kappa} S_{\kappa}(x), \quad S_{\kappa}(\widetilde{x})=\frac{1}{\sqrt{\kappa}} C_{\kappa}(x), \quad T_{\kappa}(\widetilde{x})=\frac{1}{\kappa} \frac{1}{T_{\kappa}(x)},
$$

The transformations on the cosines and sines of coordinates are derived directly from (5.2) and from the complement trigonometric relations; in some cases transfers of labels are required (yet they follow from the formalism and are not introduced by hand). We give explicitly the action on $(r, \phi)$ coordinates:

$$
\begin{gathered}
C_{\kappa_{1}}(r) \stackrel{\tau}{\longrightarrow} C_{\kappa_{1}}(\widetilde{x})=\sqrt{\kappa_{1}} S_{\kappa_{1}}(x) \\
S_{\kappa_{1}}(r) \stackrel{\tau}{\longrightarrow} S_{\kappa_{1}}(\widetilde{x})=\frac{1}{\sqrt{\kappa_{1}}} C_{\kappa_{1}}(x) \\
C_{\kappa_{2}}(\phi)=C_{\kappa_{1}}\left(\sqrt{\frac{\kappa_{2}}{\kappa_{1}}} \phi\right) \stackrel{\tau}{\longrightarrow} C_{\kappa_{1}}\left(\widetilde{\sqrt{\kappa_{2}}} v\right)=\sqrt{\kappa_{1}} S_{\kappa_{1}}\left(\sqrt{\kappa_{2}} v\right)=\sqrt{\kappa_{1} \kappa_{2}} S_{\kappa_{1} \kappa_{2}}(v) \\
S_{\kappa_{2}}(\phi)=\sqrt{\frac{\kappa_{1}}{\kappa_{2}}} S_{\kappa_{1}}\left(\sqrt{\frac{\kappa_{2}}{\kappa_{1}}} \phi\right) \stackrel{\tau}{\longrightarrow} \sqrt{\frac{\kappa_{1}}{\kappa_{2}}} S_{\kappa_{1}}\left(\sqrt{\sqrt{\kappa_{2}}} v\right)=\frac{1}{\sqrt{\kappa_{2}}} C_{\kappa_{1}}\left(\sqrt{\kappa_{2}} v\right)=\frac{1}{\sqrt{\kappa_{2}}} C_{\kappa_{1} \kappa_{2}}(v)
\end{gathered}
$$

on $(x, v)$ coordinates:

$$
\begin{aligned}
& C_{\kappa_{1} \kappa_{2}}(v)=C_{\kappa_{1}}\left(\sqrt{\kappa_{2}} v\right) \stackrel{\tau}{\longrightarrow} C_{\kappa_{1}}(\widetilde{u})=\sqrt{\kappa_{1}} S_{\kappa_{1}}(u) \\
& S_{\kappa_{1} \kappa_{2}}(v)=\frac{1}{\sqrt{\kappa_{2}}} S_{\kappa_{1}}\left(\sqrt{\kappa_{2}} v\right) \stackrel{\tau}{\longrightarrow} \frac{1}{\sqrt{\kappa_{2}}} S_{\kappa_{1}}(\widetilde{u})=\frac{1}{\sqrt{\kappa_{1} \kappa_{2}}} C_{\kappa_{1}}(u) \\
& C_{\kappa_{1}}(x) \stackrel{\tau}{\longrightarrow} C_{\kappa_{1}}\left(\sqrt{\kappa_{2}} y\right)=C_{\kappa_{1} \kappa_{2}}(y) \\
& S_{\kappa_{1}}(x) \stackrel{\sim}{\longrightarrow} S_{\kappa_{1}}\left(\sqrt{\kappa_{2}} y\right)=\sqrt{\kappa_{2}} S_{\kappa_{1} \kappa_{2}}(y)
\end{aligned}
$$

and finally on $(u, y)$ :

$$
\begin{gathered}
C_{\kappa_{1}}(u) \stackrel{\tau}{\longrightarrow} C_{\kappa_{1}}\left(\sqrt{\frac{\kappa_{2}}{\kappa_{1}}} \phi\right)=C_{\kappa_{2}}(\phi) \\
S_{\kappa_{1}}(u) \stackrel{\tau}{\longrightarrow} S_{\kappa_{1}}\left(\sqrt{\frac{\kappa_{2}}{\kappa_{1}}} \phi\right)=\sqrt{\kappa_{2}} S_{\kappa_{1} \kappa_{2}}\left(\frac{1}{\sqrt{\kappa_{1}}} \phi\right)=\sqrt{\frac{\kappa_{2}}{\kappa_{1}}} S_{\kappa_{2}}(\phi) \\
C_{\kappa_{1} \kappa_{2}}(y)=C_{\kappa_{1}}\left(\sqrt{\kappa_{2}} y\right) \stackrel{\tau}{\longrightarrow} C_{\kappa_{1}}(\widetilde{r})=\sqrt{\kappa_{1}} S_{\kappa_{1}}(r) \\
S_{\kappa_{1} \kappa_{2}}(y)=\frac{1}{\sqrt{\kappa_{2}}} S_{\kappa_{1}}\left(\sqrt{\kappa_{2}} y\right) \stackrel{\tau}{\longrightarrow} \frac{1}{\sqrt{\kappa_{2}}} S_{\kappa_{1}}(\widetilde{r})=\frac{1}{\sqrt{\kappa_{1} \kappa_{2}}} C_{\kappa_{1}}(r) .
\end{gathered}
$$


These expressions are transparent for the standard sphere, where $\kappa_{1}=1, \kappa_{2}=1$ and all are square roots of $\kappa_{1}, \kappa_{2}$ are replaced by 1: they reduce to the action on standard angular coordinates of the threefold rotation around the center of the first octant. Similarly the action of $\sigma$, which interchanges the lines $l_{1}$ and $l_{2}$, is:

$$
r \stackrel{\sigma}{\longleftrightarrow} r, \quad x \stackrel{\sigma}{\longleftrightarrow} \sqrt{\kappa_{2}} y, \quad \phi \stackrel{\sigma}{\longleftrightarrow} \widetilde{\phi}, \quad u \stackrel{\sigma}{\longleftrightarrow} \sqrt{\kappa_{2}} v,
$$

which for the sines and cosines leads to:

$$
\begin{aligned}
& C_{\kappa_{1}}(r) \stackrel{\sigma}{\longleftrightarrow} C_{\kappa_{1}}(r) \\
& C_{\kappa_{2}}(\phi) \stackrel{\sigma}{\longleftrightarrow} C_{\kappa_{2}}(\widetilde{\phi})=\sqrt{\kappa_{2}} S_{\kappa_{2}}(\phi) \quad S_{\kappa_{2}}(\phi) \stackrel{\sigma}{\longleftrightarrow} S_{\kappa_{2}}(\widetilde{\phi})=\frac{1}{\sqrt{\kappa_{2}}} C_{\kappa_{2}}(\phi) \\
& C_{\kappa_{1}}(u) \stackrel{\sigma}{\longleftrightarrow} C_{\kappa_{1}}\left(\sqrt{\kappa_{2}} v\right)=C_{\kappa_{1} \kappa_{2}}(v) \quad S_{\kappa_{1}}(u) \stackrel{\sigma}{\longleftrightarrow} S_{\kappa_{1}}\left(\sqrt{\kappa_{2}} v\right)=\sqrt{\kappa_{2}} S_{\kappa_{1} \kappa_{2}}(v) \\
& C_{\kappa_{1} \kappa_{2}}(y)=C_{\kappa_{1}}\left(\sqrt{\kappa_{2}} y\right) \stackrel{\sigma}{\longleftrightarrow} C_{\kappa_{1}}(x) \quad S_{\kappa_{1} \kappa_{2}}(y)=\frac{S_{\kappa_{1}}\left(\sqrt{\kappa_{2}} y\right)}{\sqrt{\kappa_{2}}} \stackrel{\sigma}{\longleftrightarrow} \frac{1}{\sqrt{\kappa_{2}}} S_{\kappa_{1}}(x) .
\end{aligned}
$$

In this way, as long as $\kappa_{1}, \kappa_{2}$ are positive, the transformations $\tau$ (resp. $\sigma$ ) are actual isometries belonging to $S O_{\kappa_{1}, \kappa_{2}}(3)$ (resp. to $O_{\kappa_{1}, \kappa_{2}}(3)$ ). The new triality proposal consists on considering $\tau$ and $\sigma$ in all $\kappa_{1}, \kappa_{2}$ cases. Of course these should be taken as formal transformations. However the essential point is that when the action of triality on the ambient space is coupled with the action of triality on coordinates, involving also transfer of labels all square roots disappear and the end result is meaningful again for the whole family of spaces. The perspective obtained that way helps to understand the situation for each individual space and in their relation to others.

Therefore we formally enlarge the Lie group $S O_{\kappa_{1}, \kappa_{2}}(3)$ generated by $(2.4)$ with the transformations $\tau$ and $\sigma$, with any non zero value for $\kappa_{1}, \kappa_{2}$, and we consider the group so obtained $O_{\kappa_{1}, \kappa_{2}}(3)$ as the 'full' formal group of the CK space $S_{\left[\kappa_{1}\right] \kappa_{2}}^{2}$. The transformation properties of coordinates and their trigonometric functions are defined to be described by the previous expressions. The action of these transformations on the Noether momenta is:

$$
\left(\begin{array}{c}
J \\
P_{1} \\
P_{2}
\end{array}\right) \stackrel{\tau}{\longrightarrow}\left(\begin{array}{c}
\left(-1 / \sqrt{\kappa_{1}}\right) P_{2} \\
\left(\sqrt{\kappa_{1}} / \sqrt{\kappa_{2}}\right) J \\
-\sqrt{\kappa_{2}} P_{1}
\end{array}\right), \quad\left(\begin{array}{c}
J \\
P_{1} \\
P_{2}
\end{array}\right) \stackrel{\sigma}{\rightarrow}\left(\begin{array}{c}
-J \\
\left(1 / \sqrt{\kappa_{2}}\right) P_{2} \\
\sqrt{\kappa_{2}} P_{1}
\end{array}\right) .
$$

Then it is clear that the six coordinate systems discussed in the paper lie in two different orbits under the action of the transformations $\tau$ and $\sigma$ : For the first set $\tau$ cyclically permutes the three polar, parallel ' 1 ' and parallel ' 2 ' coordinates, while $\sigma$ fixes the polar ones (with reversal on $\phi$ ) and interchanges parallel ' 1 ' and parallel ' 2 ' coordinates.

If we rename these coordinate systems using the new names polar ' 0 ' for polar, polar ' 1 ' for parallel ' 1 ' and polar ' 2 ' for parallel ' 2 ' coordinates, then the renaming highlights the similarities between the three systems.

We include a worked example going from polar ' 0 ' to polar ' 1 ' coordinates. We start from polar ' 0 ' coordinates (3.2) and apply triality by letting $\tau^{-1}$ act on the ambient space coordinates and $\tau$ on the trigonometric functions of the coordinates $(r, \phi)$ themselves. This way we arrive to $(3.3)$ in terms of the $(x, v)$ polar ' 1 ' or parallel ' 1 ' coordinates. We display this in full detail:

$$
\left(\begin{array}{c}
C_{\kappa_{1}}(r) \\
S_{\kappa_{1}}(r) C_{\kappa_{2}}(\phi) \\
S_{\kappa_{1}}(r) S_{\kappa_{2}}(\phi)
\end{array}\right) \rightarrow\left(\begin{array}{c}
\sqrt{\kappa_{1} \kappa_{2}} S_{\kappa_{1}}(r) S_{\kappa_{2}}(\phi) \\
\frac{1}{\sqrt{\kappa_{1}}} C_{\kappa_{1}}(r) \\
\frac{1}{\sqrt{\kappa_{2}}} S_{\kappa_{1}}(r) C_{\kappa_{2}}(\phi)
\end{array}\right) \rightarrow\left(\begin{array}{c}
\sqrt{\kappa_{1} \kappa_{2}} \frac{C_{\kappa_{1}}(x)}{\sqrt{\kappa_{1}}} \frac{C_{\kappa_{1} \kappa_{2}}(v)}{\sqrt{\kappa_{2}}} \\
\frac{1}{\sqrt{\kappa_{1}}} \sqrt{\kappa_{1}} S_{\kappa_{1}}(x) \\
\frac{1}{\sqrt{\kappa_{2}}} \frac{C_{\kappa_{1}}(x)}{\sqrt{\kappa_{1}}} \sqrt{\kappa_{1} \kappa_{2}} S_{\kappa_{1} \kappa_{2}}(v)
\end{array}\right)=\left(\begin{array}{c}
C_{\kappa_{1}}(x) C_{\kappa_{1} \kappa_{2}}(v) \\
S_{\kappa_{1}}(x) \\
C_{\kappa_{1}}(x) S_{\kappa_{1} \kappa_{2}}(v)
\end{array}\right)
$$


The same proccess can be performed for the polar metric going to parallel ' 1 ' metric:

$$
d r^{2}+\kappa_{2} S_{\kappa_{1}}^{2}(r) d \phi^{2} \stackrel{\tau}{\longrightarrow} d(\widetilde{x})^{2}+\kappa_{2} \frac{1}{\kappa_{1}} C_{\kappa_{1}}^{2}(x)\left(\sqrt{\frac{\kappa_{1}}{\kappa_{2}}} \sqrt{\kappa_{2}} d v\right)^{2}=d x^{2}+\kappa_{2} C_{\kappa_{1}}^{2}(x) d v^{2}
$$

and for the constant of motion (a constant $\kappa_{1}$ has been absorbed in the function $\mathcal{B}(v)$ ):

$$
J^{2}+2 \kappa_{2} \mathcal{B}(\phi) \stackrel{\tau}{\longrightarrow} \frac{1}{\kappa_{1}}\left(P_{2}^{2}+2 \kappa_{2} \mathcal{B}(v)\right)
$$

Similarly for the second set $\tau$ cyclically permutes the three equiparabolic ' 12 ', equiparabolic ' 20 ' and equiparabolic ' 01 ', while $\sigma$ fixes the equiparabolic ' 12 ' ones (with interchange on $\left(z_{+}, z_{-}\right)$) and exchanges equiparabolic ' 01 ' to equiparabolic '20' coordinates. In this case the names suggesting triality have been introduced from the beginning.

This $T$-symmetry underlies the analogies between the three coordinate systems within each set. All results concerning each individual coordinate system could have been derived by using the $T$-replacement rules from any other in the same set. Indeed the paper has been written as to implicitly stress this symmetry from the beginning. Within this wiewpoint, polar ' 0 ', with constants of type $J^{2}$ and equiparabolic ' 12 ', with constant of type $P_{1} P_{2}$ might be considered as the truly basic coordinate systems amongst these six as they are invariant under $\sigma$ and generate under $\tau$ the complete set. The action of the $T$-symmetry allows to obtain the remaining coordinates starting from them. This $T$-symmetry plays an essential role to simplify the general study of "elliptic" coordinates on $S_{\left[\kappa_{1}\right] \kappa_{2}}^{2}$.

\section{Two examples: Harmonic oscillator and Kepler potentials in $S_{\left[\kappa_{1}\right] \kappa_{2}}^{2}$}

The harmonic oscillator $V=\frac{1}{2} \omega_{0}^{2} r^{2}$ and the Kepler potential $V=-k / r$ are distinguished in $\mathbf{E}^{3}$ (and in $\mathbf{E}^{2}$ ). All the properties responsible for this distinguished character are linked to their superintegrability. Now we show that this property is generic from the CK viewpoint, i.e., there are $\kappa_{1}, \kappa_{2}$ "curved" versions of both harmonic oscillator and Kepler potentials which keep the superintegrable character for all values of $\kappa_{1}, \kappa_{2}$. The outstanding properties of these Euclidean potentials are indeed generic for the harmonic oscillator and the Kepler potentials in any $S_{\left[\kappa_{1}\right] \kappa_{2}}^{2}$.

\subsection{The harmonic oscillator in curved spaces}

In any non degenerate space of constant curvature the "harmonic oscillator" potential is defined to be:

$$
V_{H O}=\frac{1}{2} \omega_{0}^{2} T_{\kappa_{1}}^{2}(r)
$$

Polar coordinates are natural to study this potential because the invariance of $V_{H O}$ under rotations around the potential center leads to constancy of the angular momentum. Trivially then there is a quadratic constant $I=J^{2}$. In the standard sphere $\mathbf{S}^{2}$ this potential was first studied by Higgs [11] and Leemon [15].

Now in any $S_{\left[\kappa_{1}\right] \kappa_{2}}^{2}$, trigonometric relations allow one to conclude the identities 


$$
\begin{aligned}
T_{\kappa_{1}} 2(r) & =\frac{T_{\kappa_{1}} 2(u)}{C_{\kappa_{1} \kappa_{2}} 2(y)}+\kappa_{2} T_{\kappa_{1} \kappa_{2}} 2(y)=T_{\kappa_{1}} 2(x)+\kappa_{2} \frac{T_{\kappa_{1} \kappa_{2}} 2(v)}{C_{\kappa_{1}} 2(x)} \\
& =\frac{1}{2 C_{\kappa_{1}}(x) C_{\kappa_{1} \kappa_{2}}(y)}\left\{\frac{S_{\kappa_{1}} 2\left(2 z_{+}\right)}{C_{\kappa_{1}}\left(2 z_{+}\right)}+\frac{S_{\kappa_{1}} 2\left(2 z_{-}\right)}{C_{\kappa_{1}}\left(2 z_{-}\right)}\right\},
\end{aligned}
$$

displaying the separable nature of the harmonic oscillator not only in polar, but also in parallel ' 1 ' and ' 2 ' and equiparabolic ' 12 ' coordinates as well. In parallel ' 1 ' the same potential has again the separable form (4.10), with a constant of motion (4.11):

$$
\mathcal{A}(x)=\frac{1}{2} \omega_{0}^{2} S_{\kappa_{1}}^{2}(x), \quad \mathcal{B}(v)=\frac{1}{2} \omega_{0}^{2} \kappa_{2} T_{\kappa_{1} \kappa_{2}}^{2}(v), \quad 2 I_{P_{2}^{2}}=P_{2}^{2}+\kappa_{2}^{2} \omega_{0}^{2} T_{\kappa_{1} \kappa_{2}}{ }^{2}(v) .
$$

Analogously, in parallel '2' $V_{H O}$ has the separable form with functions $\mathcal{A}, \mathcal{B}(4.12)$ and constant of motion $I$ (4.13) given by:

$$
\mathcal{A}(y)=\frac{1}{2} \omega_{0}^{2} \kappa_{2} S_{\kappa_{1} \kappa_{2}}^{2}(y), \quad \mathcal{B}(u)=\frac{1}{2} \omega_{0}^{2} T_{\kappa_{1}}^{2}(u), \quad 2 I_{P_{1}^{2}}=\kappa_{2} P_{1}^{2}+\kappa_{2} \omega_{0}^{2} T_{\kappa_{1}}^{2}(u) .
$$

Finally in equiparabolic ' 12 ' coordinates the harmonic oscillator potential has also separable form (4.14), with:

$$
\mathcal{A}\left(z_{+}\right)=\frac{1}{2} \omega_{0}^{2} T_{\kappa_{1}}\left(2 z_{+}\right) S_{\kappa_{1}}\left(2 z_{+}\right), \quad \mathcal{B}\left(z_{-}\right)=\frac{1}{2} \omega_{0}^{2} T_{\kappa_{1}}\left(2 z_{-}\right) S_{\kappa_{1}}\left(2 z_{-}\right),
$$

and has the constant of motion (4.15)

$$
I_{P_{1} P_{2}}=-\sqrt{\kappa_{2}} P_{1} P_{2}-\kappa_{2} \sqrt{\kappa_{2}} \omega_{0}^{2} \frac{S_{\kappa_{1}}(x) S_{\kappa_{1} \kappa_{2}}(y)}{C_{\kappa_{1}}\left(2 z_{+}\right) C_{\kappa_{1}}\left(2 z_{-}\right)} .
$$

Taken altogether the constants $I_{P_{1}^{2}}, I_{P_{2}^{2}}, I_{P_{1} P_{2}}$ are the components of the Fradkin tensor in any space $S_{\left[\kappa_{1}\right] \kappa_{2}}^{2}$; in the standard Euclidean $\mathbf{E}^{2}$, taking into account of the accidental coincidences $u=x, v=y$, these constants reduce as they should to:

$$
\left.I_{P_{1}^{2}}\right|_{\mathbf{E}^{2}}=\frac{1}{2}\left(P_{1}^{2}+\omega_{0}^{2} x^{2}\right),\left.\quad I_{P_{2}^{2}}\right|_{\mathbf{E}^{2}}=\frac{1}{2}\left(P_{2}^{2}+\omega_{0}^{2} y^{2}\right),\left.\quad I_{P_{1} P_{2}}\right|_{\mathbf{E}^{2}}=-P_{1} P_{2}-\omega_{0}^{2} x y .
$$

Thus the essential property of the Euclidean harmonic oscillator, to have a tensor constant of motion, survives for any $\kappa_{1}, \kappa_{2}$. Note, however, that we have obtained the simplest expressions for each component of this tensor, which are referred to different ccordinate systems; to make meaningful use of this $\kappa_{1}, \kappa_{2}$-Fradkin tensor one must express all their components in a common coordinate system. The equiparabolic '12' offers the more symmetric expressions. Thus the curved harmonic oscillator is superintegrable in any $S_{\left[\kappa_{1}\right] \kappa_{2}}^{2}$.

\subsection{The Kepler potential in curved spaces}

In any $S_{\left[\kappa_{1}\right] \kappa_{2}}^{2}$ the Kepler potential is defined to be:

$$
V_{K}=-k / T_{\kappa_{1}}(r) \text {. }
$$

As in the harmonic oscillator case, invariance of $V_{K}$ under rotations around the potential center leads to constancy of angular momentum and hence there is a quadratic constant $I=J^{2}$. On the sphere this potential was first introduced by Schrödinger [29]. 
Now in any $S_{\left[\kappa_{1}\right] \kappa_{2}}^{2}$, the Kepler potential turns out to be separable also in the equiparabolic ' 01 ' and ' 20 ' coordinate systems. It is not separable in the remaining three systems.

In the equiparabolic ' 20 ' system the Kepler potential has separable form (4.16), with functions $\mathcal{A}, \mathcal{B}$ and constant of motion $I$ (4.17) given by:

$$
\mathcal{A}\left(b_{-}\right)=-k C_{\kappa_{1}}\left(2 b_{-}\right), \quad \mathcal{B}\left(b_{+}\right)=-k C_{\kappa_{1}}\left(2 b_{+}\right), \quad I_{J P_{1}}=\sqrt{\kappa_{2}} J P_{1}+\kappa_{2} \sqrt{\kappa_{2}} k S_{\kappa_{2}}(\phi) .
$$

Analogously in equiparabolic '01' coordinates the Kepler potential has separable form (4.18) with functions $\mathcal{A}, \mathcal{B}$ and constant of motion $I$ (4.19) given by:

$$
\mathcal{A}\left(a_{-}\right)=-k C_{\kappa_{1}}\left(2 a_{-}\right), \quad \mathcal{B}\left(a_{+}\right)=-k C_{\kappa_{1}}\left(2 a_{+}\right), \quad I_{J P_{2}}=-J P_{2}+\kappa_{2} k C_{\kappa_{2}}(\phi) .
$$

The constants $I_{J P_{2}}, I_{J P_{1}}$, considered altogether, must be seen as the components of the Laplace-Runge-Lenz vector in any space $S_{\left[\kappa_{1}\right] \kappa_{2}}^{2}$; in the standard Euclidean $\mathbf{E}^{2}$, these constants reduce to:

$$
\left.I_{J P_{2}}\right|_{\mathbf{E}^{2}}=-J P_{2}+k \cos \phi,\left.\quad I_{J P_{1}}\right|_{\mathbf{E}^{2}}=J P_{1}+k \sin \phi .
$$

Thus the essential property of the Kepler potential, to have an extra vector constant of motion, survives for any $\kappa_{1}, \kappa_{2}$. In this case the natural coordinates to take advantage of the existence of the Laplace-Runge-Lenz vector are polar ones.

We have therefore proven the superintegrability of the Kepler potential in any $S_{\left[\kappa_{1}\right] \kappa_{2}}^{2}$.

\section{Acknowledgments.}

We acknowledge $P$. Leach for his very careful reading and suggestions. Support of projects BFM-2003-02532, FPA-2003-02948, BFM-2002-03773 and CO2-399 is acknowledged.

\section{References}

[1] Benenti S, Intrinsic characterization of the variable separation in the Hamilton-Jacobi equation, J. Math. Phys. 38 (1997), 6578-6602.

[2] Crampin M, Conformal Killing tensors with vanishing torsion and the separation of variables in the Hamilton-Jacobi equation, Diff. Geom. Appl. 18 (2003), 87-102.

[3] Fris T I, Mandrosov V, Smorodinsky V A, Uhlir M and Winternitz P, On higher symmetries in quantum mechanics, Phys. Lett. 16 (1965), 354-356.

[4] Grosche C, The path integral for the Kepler problem on the pseudosphere, Ann. Phys. 204 (1990), 208-222.

[5] Grosche C, On the path integral in imaginary Lobachevsky space, J. Phys. A: Math. Gen. 27 (1994), 3475-3489.

[6] Grosche C, Pogosyan G S and Sissakian A N, Path integral discussion for SmorodinskyWinternitz potentials I, Fortschr. Phys. 43 (1995), 453-521.

[7] Grosche C, Pogosyan G S and Sissakian A N, Path integral discussion for SmorodinskyWinternitz potentials II, Fortschr. Phys. 43 (1995), 523-563.

[8] Herranz F J, Ortega R and Santander M, Trigonometry of space-times: a new self-dual approach to a curvature/signature (in)dependent trigonometry, J. Phys. A: Math. Gen. 33 (2000), 4525-4551. 
[9] Herranz F J and Santander M, Conformal symmetries of space-times, J. Phys. A: Math. Gen. 35 (2002), 6601-6618.

[10] Herranz F J and Santander M, Conformal compactification of space-times, J. Phys. A: Math. Gen. 35 (2002), 6619-6629.

[11] Higgs P W, Dynamical symmetries in a spherical geometry I, J. Phys. A: Math. Gen. 12 (1979), 309-323.

[12] Kalnins E G, On the separation of variables of the Laplace equation in two and three dimensional Minkowski space, SIAM J. Math. Anal., 6 (1975), 340-374.

[13] Kalnins E G, Kress J M, Pogosyan G S and Miller W, Completeness of superintegrability in two-dimensional constant-curvature spaces, J. Phys. A: Math. Gen. 34 (1979), 4705-4720 (2001).

[14] Kalnins E G, Kress J M and Winternitz P, Superintegrability in a two-dimensional space of nonconstant curvature, J. Math. Phys. 43 (2002), 970-983.

[15] Leemon H I, Dynamical symmetries in a spherical geometry II, J. Phys. A: Math. Gen. 12 (1979), 489-501.

[16] McLenaghan R G and Smirnov R G, Intrinsic Characterization of Orthogonal Separability for Natural Hamiltonians with Scalar potentials on Pseudo-Riemannian Spaces, J. Nonlinear Math. Phys., 9 (2002), 140-151.

[17] McLenaghan R G, Smirnov R G and The D, An extension of the classical theory of algebraic invariants to pseudo-Riemannian geometry and Hamiltonian mechanics, J. Math. Phys. 45 (2004), 1079-1120.

[18] Marmo G, Saletan E, Simoni A and Vitale B, Dynamical Systems: A Differential Geometric Approach to Symmetry and Reduction, Wiley, Chichester, 1985.

[19] Olevski M N , Triorthogonal systems in spaces of constant curvature in which the equation $\Delta_{3} u+\lambda u=0$ allows a complete separation of variables, Mat. Sb., 27 (1950), 379-426 (In Russian).

[20] Rañada M F , Superintegrable $n=2$ systems, quadratic constants of motion, and potentials of Drach, J. Math. Phys. 38 (1997), 4165-4178.

[21] Rañada M F and Santander M, Superintegrable systems on the two-dimensional sphere $S^{2}$ and the hyperbolic plane $H^{2}$, J. Math. Phys. 40 (1999), 5026-5057.

[22] Rañada M F and Santander M, Complex Euclidean superintegrable potentials, potentials of Drach, and potential of Holt, Phys. Lett. A 278 (2001), 271-279.

[23] Rañada M F and Santander M, On some properties of harmonic oscillator on spaces of constant curvature, Rep. Math. Phys. 49 (2002), 335-343.

[24] Rañada M F and Santander M, On the Harmonic Oscillator on the two-dimensional sphere $S^{2}$ and the hyperbolic plane $H^{2}, J$. Math. Phys. 43 (2002), 431-451.

[25] Rañada M F and Santander M, On the Harmonic Oscillator on the two-dimensional sphere $S^{2}$ and the hyperbolic plane $H^{2}$ II, J. Math. Phys. 44 (2003), 2149-2167.

[26] Rañada M F, Sanz-Gil T and Santander M, Superintegrable potentials and superposition of Higgs oscillators on the sphere $S^{2}$, in Classical and Quantum Integrability, Banach Center Publications Vol. 59 (2003), 243-255.

[27] Sanz-Gil T, Superintegrability in spaces of constant curvature, In preparation. 
[28] Santander M, The Hyperbolic-AntiDeSitter-DeSitter triality, in Proceedings of the Meeting Lorentzian Geometry (Benalmádena, Spain), Cañadas-Pinedo M A, Gutiérrez M and Romero A Eds., Publications of the RSME, 5 (2003), 247-260.

[29] Schroedinger E, A method of determining quantum mechanical eigenvalues and eigenfunctions, Proc. R.I.A. A 46 (1940), 9-16.

[30] Slawianowski J J, Bertrand systems on spaces of constant sectional curvature, Rep. Math. Phys. 46 (2000), 429-460. 